\title{
Intramolecular Arene Epoxidation by Phosphadioxiranes
}

\author{
Dong Zhang, ${ }^{\dagger}$ Ruomei Gao, ${ }^{\dagger}$ Shabana Afzal, ${ }^{\dagger}$ Mario Vargas, ${ }^{\dagger}$ Shantanu Sharma, \\ Alison McCurdy, ${ }^{\dagger}$ Muhammed Yousufuddin,,${ }^{\S}$ Timothy Stewart, ${ }^{\S}$ Robert Bau, ${ }^{\S}$ and \\ Matthias Selke*, \\ ${ }^{\dagger}$ Department of Chemistry and Biochemistry, California State University, Los Angeles, \\ Los Angeles, CA, 90032 \\ ${ }^{\ddagger}$ Department of Chemistry and Center for Macromolecular Modeling and Materials \\ Design, California State Polytechnic University, Pomona, \\ Pomona, CA, 91768 \\ ${ }^{\S}$ Department of Chemistry, University of Southern California, Los Angeles, CA 90089 \\ mselke@calstatela.edu
}

\section{Supporting Information}

\section{General information:}

All reagents were obtained commercially and used without further purification unless stated otherwise. NMR spectra were acquired in deuterated toluene on a Bruker AM300 at $25{ }^{\circ} \mathrm{C}$, if not stated otherwise ${ }^{1} \mathrm{H}$ NMR spectra were recorded at $300 \mathrm{MHz},{ }^{31} \mathrm{P}$ NMR spectra were recorded at $121 \mathrm{MHz}$, Chemical shifts in NMR spectra are expressed in ppm, and all coupling constants are expressed in Hz. CD spectra were carried out using a JASCO J-810 spectropolarimeter, with $1 \mathrm{~mm}$ cell in freshly prepared acetonitrile solututions at room temperature.

Photooxidations were carried out in oxygen-saturated deuterated toluene solutions. Typically, $0.8 \mathrm{~mL}$ of the solution of the substrate $(20 \mathrm{mM})$ and the photosensitizer $(5$, 10, 15, 20-Tetraphenyl-21H, 23H-porphine, TPP, $10^{-6} \mathrm{M}$ ) in a NMR tube were irradiated by a $200 \mathrm{~W}$ Oriel tungsten-halogen lamp. A glass filter was employed to cut off light below $498 \mathrm{~nm}$. The oxidized products were monitored and directly analyzed 
by ${ }^{1} \mathrm{H}$ NMR and ${ }^{31} \mathrm{P}$ NMR.

\section{Detection of the phosphadioxiane $\left({ }^{31}\right.$ P NMR Spectra)}

Photooxidations of 1,1'-binaphthyl di-t-butyl phosphine (1) were carried out in NMR tubes kept in an acetonitrile slush bath at $-40{ }^{\circ} \mathrm{C}$ in a transparent dewar using an $200 \mathrm{~W}$ Oriel tungsten-halogen lamp equipped with water filter and cutoff filter at $498 \mathrm{~nm}$. The concentration of 1,1'-binaphthyl di-t-butyl phosphine (1) was ca. $20 \mathrm{mM}$ in toluene at $-40{ }^{\circ} \mathrm{C}$; The concentration of TPP was ca. $10^{-6} \mathrm{M} .{ }^{31} \mathrm{P}$ NMR spectra were recorded after 10 min irradiation of starting material 1 at $-40{ }^{\circ} \mathrm{C}$ in deuterated toluene. ${ }^{31} \mathrm{P}$ NMR spectrum of the irradiated sample $\left(-40^{\circ} \mathrm{C}\right)$ shows phosphadioxirane $4, \delta$ : $-18.6 \mathrm{ppm}$, phosphine oxide 3, $\delta: 50.8 \mathrm{ppm}$ and starting phosphine 1, $\delta: 17.7 \mathrm{ppm}$

${ }^{31} \mathrm{P}$ NMR $\left(121 \mathrm{MHz}\right.$, Toluene- $\left.\mathrm{d}_{8}\right),-40^{\circ} \mathrm{C}$

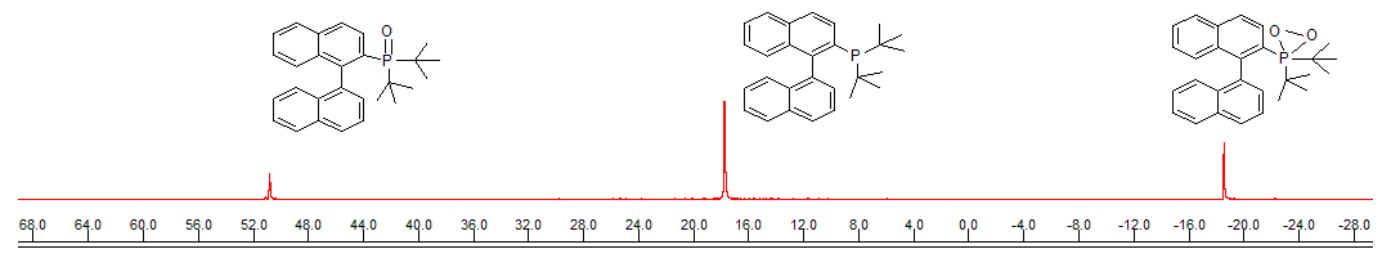

The above reaction mixture was warmed to R. T. for $30 \mathrm{~min}$, then cooled to $-40{ }^{\circ} \mathrm{C}$, shows the new peak of compound 2, $\delta: 51.1 \mathrm{ppm}$, and that the peak of phosphadioxirane $\mathbf{4}$ disappeared.

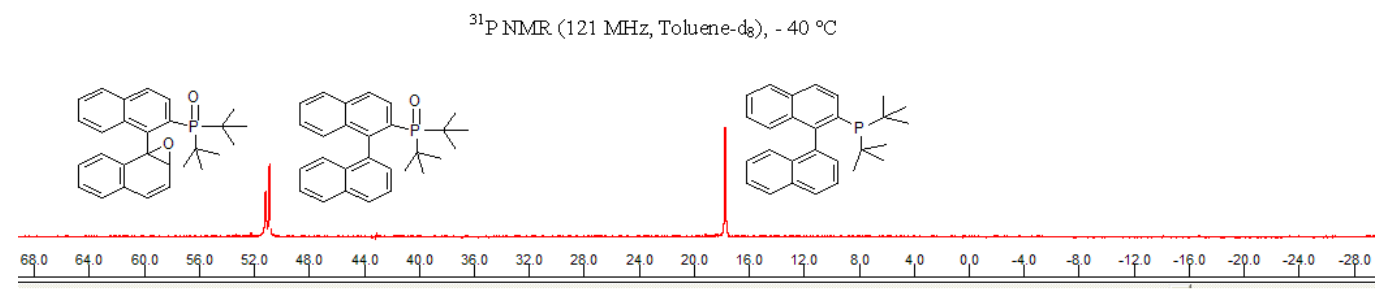




\section{${ }^{1}$ H NMR of Products $2 \& 3$ at room temperature}

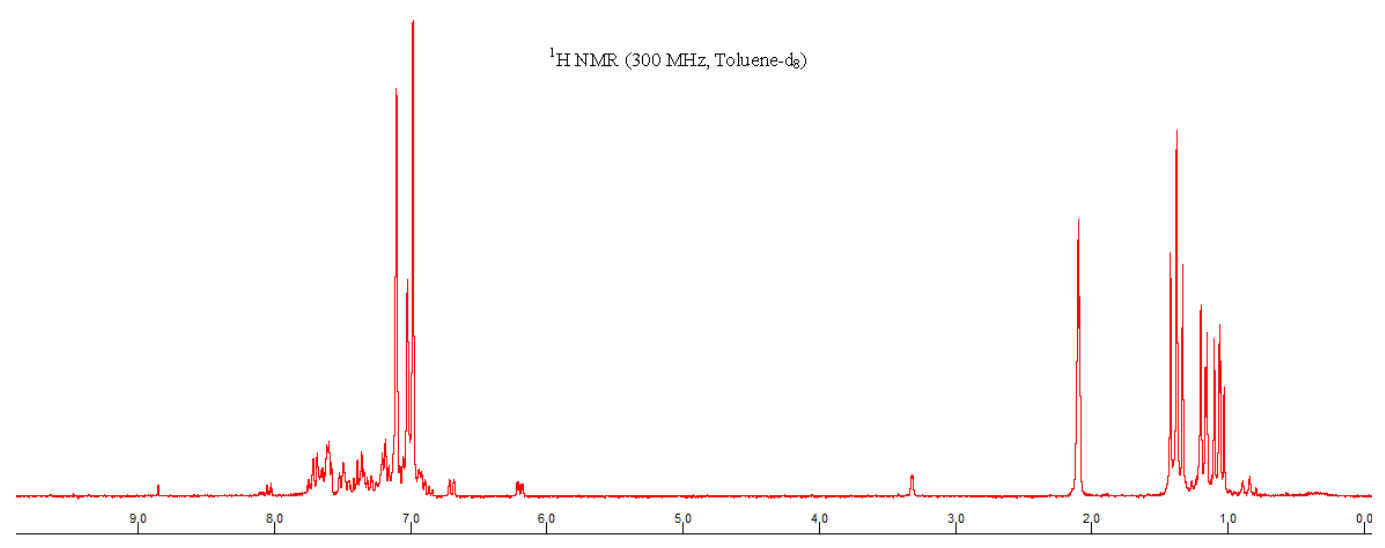

Product 2, ${ }^{1} \mathrm{H}$ NMR (300 MHz, Toluene- $\left.\mathrm{d}_{8}\right) \delta: 6.80-9.00$ (m, aromatic protons, $10 \mathrm{H}$ ), $6.69(\mathrm{~d}, J=9.7 \mathrm{~Hz}, 1 \mathrm{H}), 6.19(\mathrm{dd}, J=3.6$ and $9.6 \mathrm{~Hz}, 1 \mathrm{H}), 3.31(\mathrm{~m}, 1 \mathrm{H}), 1.39$ (d, $J=$ $14.3 \mathrm{~Hz}, 9 \mathrm{H}), 1.34(\mathrm{~d}, J=13.2 \mathrm{~Hz}, 9 \mathrm{H})$.

Product 3, ${ }^{1} \mathrm{H}$ NMR (300 MHz, Toluene- $\left.\mathrm{d}_{8}\right) \delta$ : 6.80-9.00 (m, aromatic protons, $13 \mathrm{H}$ ), $1.16(\mathrm{~d}, J=13.0 \mathrm{~Hz}, 9 \mathrm{H}), 1.07(\mathrm{~d}, J=13.0 \mathrm{~Hz}, 9 \mathrm{H})$.

\section{NMR spectra of compound 5}

${ }^{31}$ P NMR (121 MHz, Toluene-d 8 ), $\delta: 57.8$ ppm

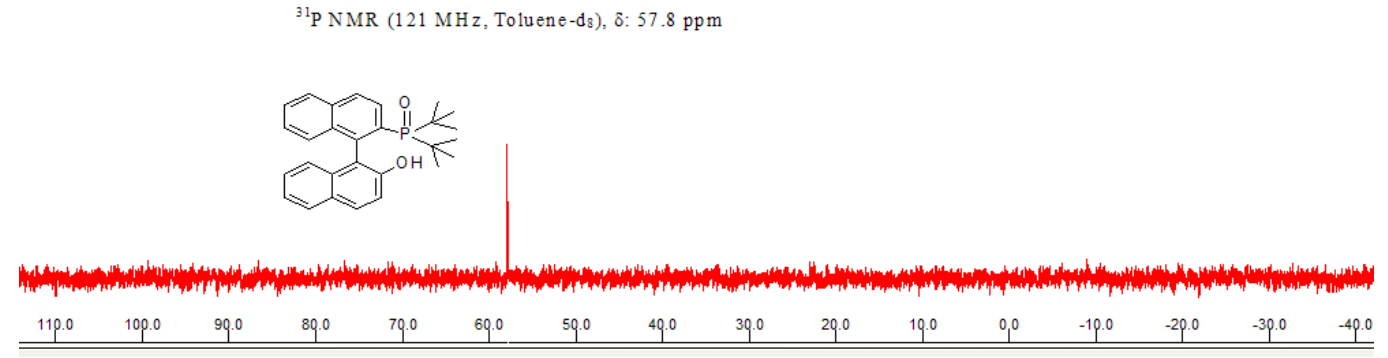

${ }^{1} \mathrm{H}$ NMR (300 MHz, Toluene-d 8 ) $\delta: 6.50-9.20$ (m, aromatic protons, $\left.12 \mathrm{H}\right), 1.57(\mathrm{~s}, 1 \mathrm{H})$, $1.21(\mathrm{~d}, J=13.0 \mathrm{~Hz}, 9 \mathrm{H}), 0.93(\mathrm{~d}, J=14.0 \mathrm{~Hz}, 9 \mathrm{H})$ 


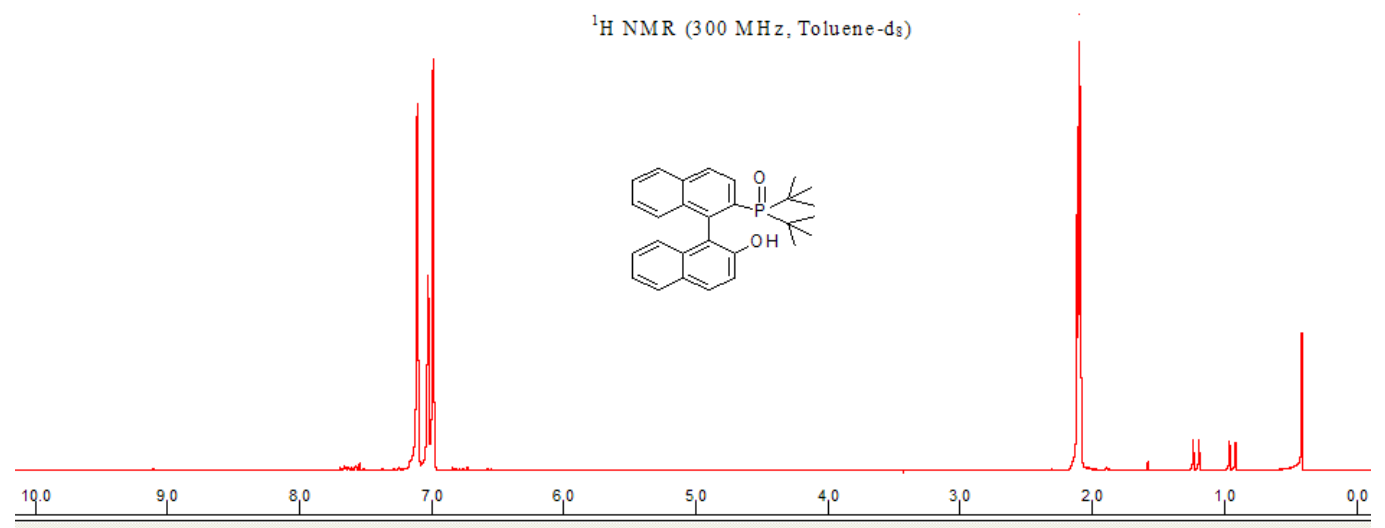

\section{NMR spectra of product 7}

${ }^{31}$ P NMR (121 MHz, Toluene-d 8 ), $\delta: 27.9$ ppm

${ }^{31}$ P NMR (121 MHz, Toluene-d 8 ), $8: 27.9 \mathrm{ppm}$

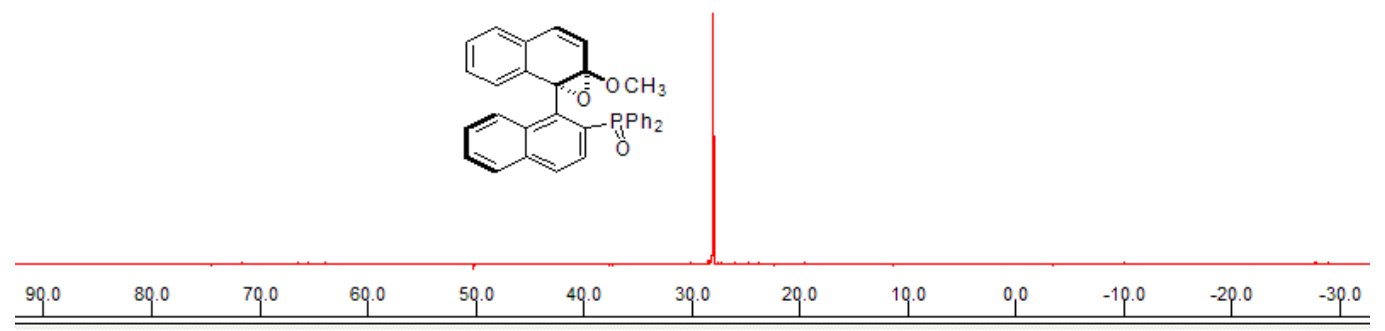

${ }^{1}$ H NMR (300 MHz, Toluene-d 8 ), $\delta: 6.60-9.00$ (m, aromatic protons, $\left.12 \mathrm{H}\right), 6.57(\mathrm{~d}, J=$ $9.9 \mathrm{~Hz}, 1 \mathrm{H}), 6.27(\mathrm{~d}, J=9.8 \mathrm{~Hz}, 1 \mathrm{H}), 2.60(\mathrm{~s}, 3 \mathrm{H})$

${ }^{1}$ H NMR (300 MH $z$, Toluene- $\left.d_{8}\right)$

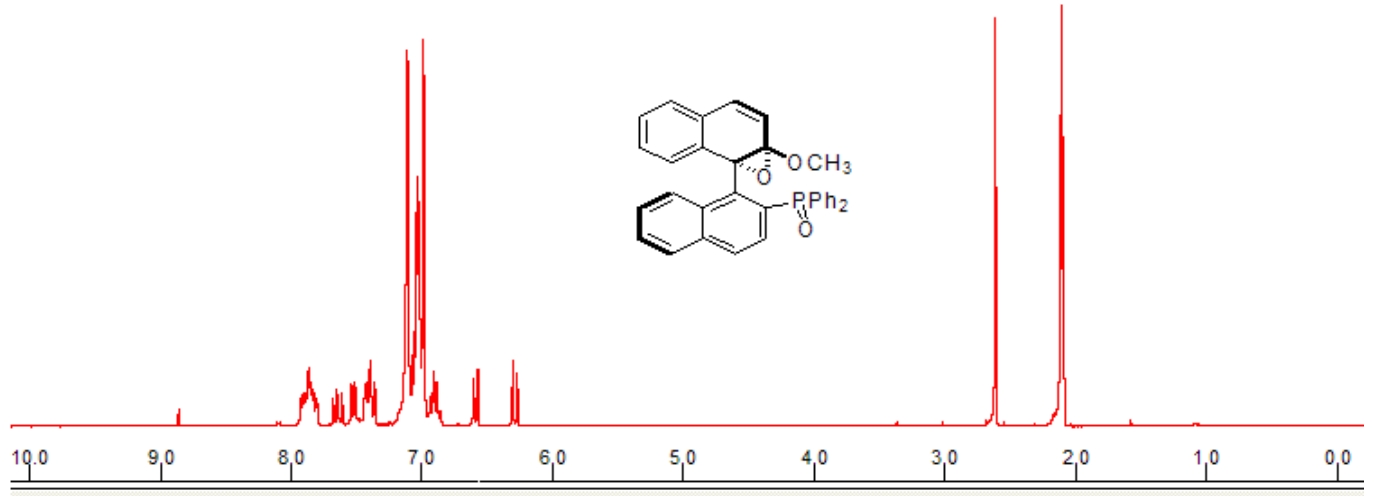

All the ${ }^{1} \mathrm{H}$ NMR and ${ }^{31} \mathrm{P}$ NMR spectra of $7 S$-isomer are identical with $7 R$-isomer. 


\section{CD spectra:}

$\mathrm{CD}$ spectra were taken in acetonitrile solutions of compound 6 and $7\left(1.6 \times 10^{-4} \mathrm{M}\right)$. We used methylene blue as the sensitizer and during the irradiation the concentration of the solution remained constant.
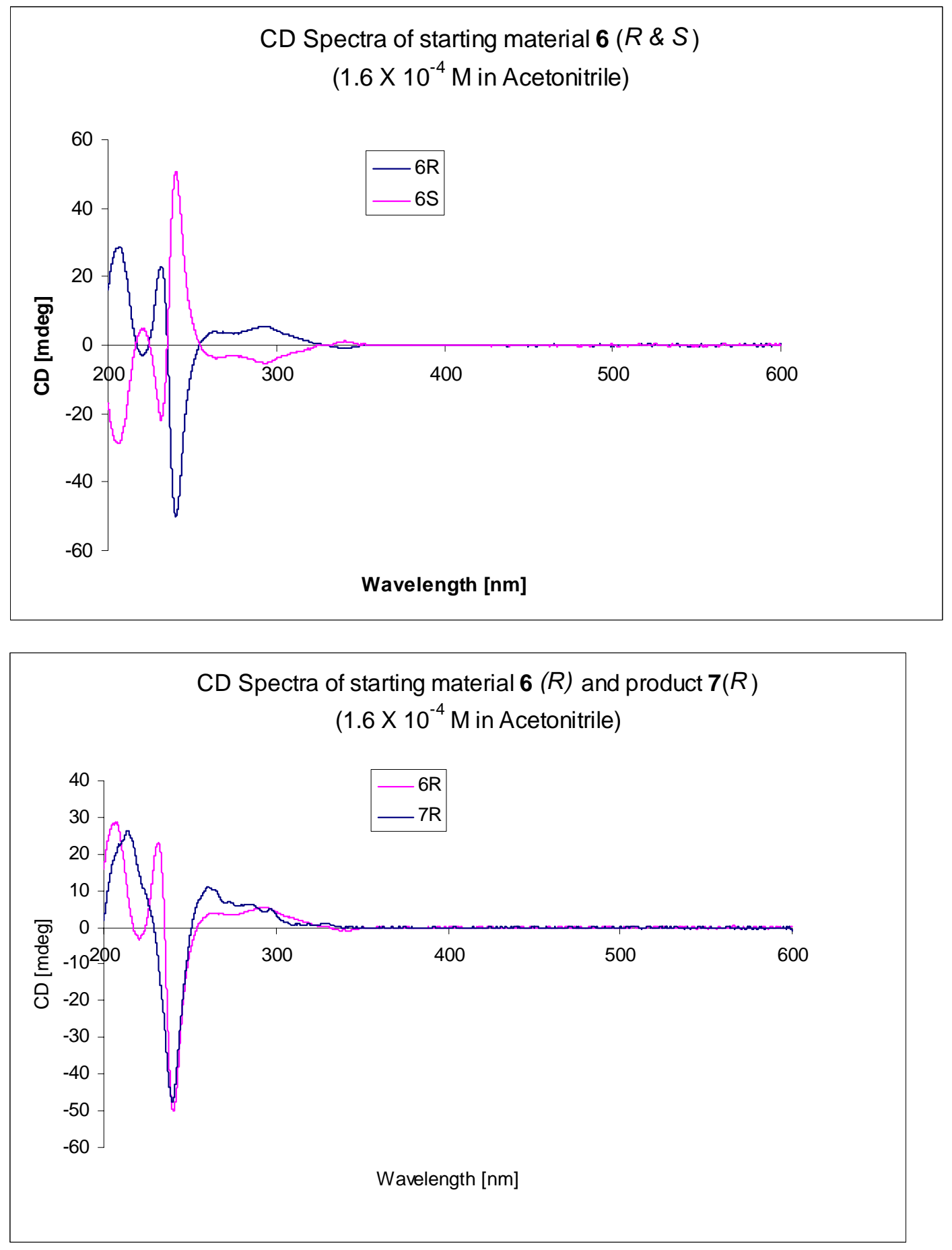


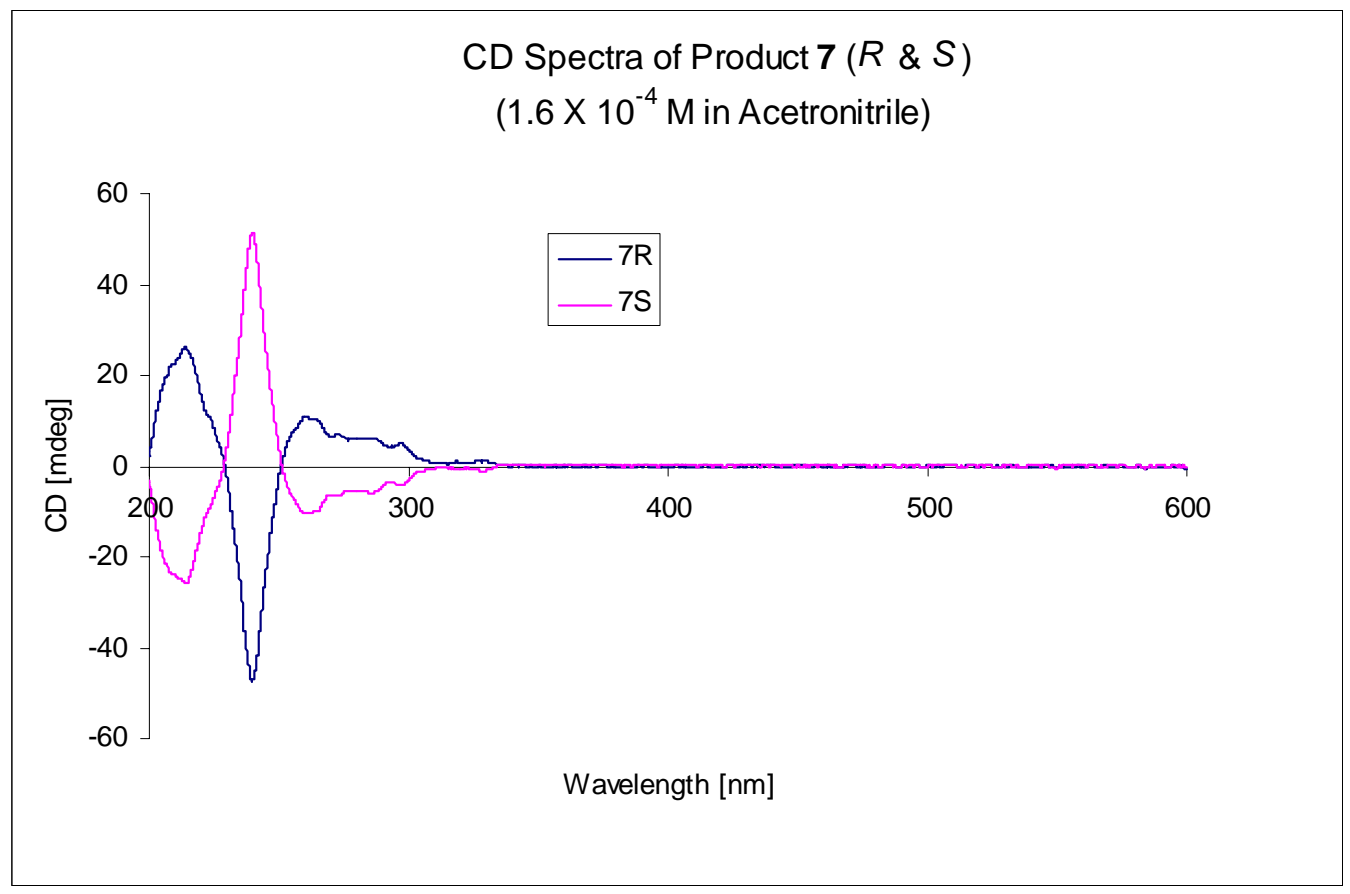

CD data:

(R)-2-(diphenylphosphino)-2'-methoxy-1,1'-binaphthyl, $\mathbf{6}(R), \Delta \varepsilon_{220}-6.1, \Delta \varepsilon_{232}+43.6$, $\Delta \varepsilon_{240}-94.6$.

(S)-2-(diphenylphosphino)-2'-methoxy-1,1'-binaphthyl, $6(S), \quad \Delta \varepsilon_{220}+9.0, \quad \Delta \varepsilon_{232}-41.5$, $\Delta \varepsilon_{240}+95.8$

(R)-2-(diphenylphosphine $\quad$ oxide)-2'-methoxy-2'-epoxy-1,1'-binaphthyl, $\quad$ 7 $(R)$, $\Delta \varepsilon_{214}+49.9, \Delta \varepsilon_{240}-89.9$

(S)-2-(diphenylphosphine $\quad$ oxide)-2'-methoxy-2'-epoxy-1,1'-binaphthyl, $\quad$ 7(S), $\Delta \varepsilon_{214}-49.2, \Delta \varepsilon_{240}+97.1$ 


\section{X-Ray Data of Compound 2}

X-ray diffraction data from a crystal of $\mathbf{2}$ obtained by crystallization from acetonitrile at $-20{ }^{\circ} \mathrm{C}$ were collected at $130 \mathrm{~K}$ on a SMART APEX CCD diffractometer with graphite fine-focused monochromatic Mo-K $\alpha$ radiation $(\lambda=0.71073 \AA)$. The cell parameters for 2 were obtained from the least-squares refinement of the spots (from 60 collected frames) using the SMART program. A hemisphere of the $0.3 \times 0.2 \times 0.5 \mathrm{~mm}^{3}$ phosphorous crystal complex were collected up to a resolution of $0.75 \AA$, the intensity data were processed using the Saint Plus program. All calculations for the structure determination were carried out using the SHELXTL package (version 6.14) [1]. Initial atomic position were located by direct methods using XS, and the structure was refined by the least square methods using SHELX with 3759 independent reflections and within the range of theta $1.92-23.27^{\circ}$ (completeness $99.8 \%$ ). Calculated hydrogen position were input and refined in a riding manner along with the corresponding carbons. A summary of the refinement details and the resulting factors are given in Table 1-5. This structure represents, to the best of our knowledge by searching the Cambridge Structural Database, the first time a naphthalene epoxide complex has been characterized by X-ray crystallography.

Table 1. Crystal data and structure refinement for $\left(\mathrm{P}_{1} \mathrm{O}_{2} \mathrm{C}_{28} \mathrm{H}_{30}\right) \cdot\left(\mathrm{N}_{1} \mathrm{C}_{2} \mathrm{H}_{3}\right)$ (compound 2).

Empirical formula

Formula weight

Temperature

Wavelength

Crystal system

Space group

Unit cell dimensions

Volume

$\mathrm{Z}$

Density (calculated)

Absorption coefficient

$\mathrm{F}(000)$
$\mathrm{C} 30 \mathrm{H} 24 \mathrm{~N} \mathrm{O} 2 \mathrm{P}$

461.47

130(2) K

$0.71073 \AA$

monoclinic

$\mathrm{P} 2(1) / \mathrm{n}$

$\mathrm{a}=10.999(6) \AA$

$\alpha=90^{\circ}$.

$\mathrm{b}=11.201(6) \AA$

$\beta=100.571(9)^{\circ}$.

$\mathrm{c}=21.538(12) \AA$

$\gamma=90^{\circ}$.

2609(2) $\AA^{3}$

4

$1.175 \mathrm{Mg} / \mathrm{m}^{3}$

$0.131 \mathrm{~mm}^{-1}$

968 
Crystal size

Theta range for data collection

Index ranges

Reflections collected

Independent reflections

Completeness to theta $=23.27^{\circ}$

Refinement method

Data / restraints / parameters

Goodness-of-fit on $\mathrm{F}^{2}$

Final $\mathrm{R}$ indices [I $>2 \operatorname{sigma}(\mathrm{I})]$

$\mathrm{R}$ indices (all data)

Largest diff. peak and hole
$0.3 \times 0.2 \times 0.05 \mathrm{~mm}^{3}$

1.92 to $23.27^{\circ}$.

$-11<=\mathrm{h}<=12,-12<=\mathrm{k}<=10,-23<=1<=23$

11192

$3759[\mathrm{R}(\mathrm{int})=0.0488]$

$99.8 \%$

Full-matrix least-squares on $\mathrm{F}^{2}$

$3759 / 20 / 341$

1.060

$\mathrm{R} 1=0.0777, \mathrm{wR} 2=0.2286$

$\mathrm{R} 1=0.0974, \mathrm{wR} 2=0.2424$

0.635 and -0.292 e. $\AA^{-3}$ 
Table 2. Atomic coordinates ( $\left.\mathrm{x} 10^{4}\right)$ and equivalent isotropic displacement parameters $\left(\AA^{2} \mathrm{x}\right.$ $10^{3}$ ) for $\left(\mathrm{P}_{1} \mathrm{O}_{2} \mathrm{C}_{28} \mathrm{H}_{30}\right) \cdot\left(\mathrm{N}_{1} \mathrm{C}_{2} \mathrm{H}_{3}\right)$ (compound 2). $\mathrm{U}(\mathrm{eq})$ is defined as one third of the trace of the orthogonalized $\mathrm{U}^{\mathrm{ij}}$ tensor.

\begin{tabular}{|c|c|c|c|c|}
\hline & $\mathrm{x}$ & $\mathrm{y}$ & $\mathrm{z}$ & $\mathrm{U}(\mathrm{eq})$ \\
\hline $\mathrm{P}(1)$ & $8581(1)$ & $7926(1)$ & $1342(1)$ & $41(1)$ \\
\hline $\mathrm{N}(1)$ & 2801(5) & 7471(5) & $-184(2)$ & $89(2)$ \\
\hline $\mathrm{O}(1)$ & $9196(2)$ & $8670(3)$ & $922(1)$ & $45(1)$ \\
\hline $\mathrm{O}(2)$ & 11543(3) & $8404(3)$ & $1600(1)$ & $54(1)$ \\
\hline $\mathrm{C}(1)$ & $9167(4)$ & $8235(4)$ & 2182(2) & $45(1)$ \\
\hline $\mathrm{C}(2)$ & $8531(4)$ & 7751(4) & 2632(2) & $47(1)$ \\
\hline$C(3)$ & $8846(4)$ & $8000(4)$ & $3258(2)$ & $48(1)$ \\
\hline $\mathrm{C}(4)$ & $9789(4)$ & $8817(4)$ & $3479(2)$ & $42(1)$ \\
\hline $\mathrm{C}(5)$ & 10105(4) & 9143(5) & $4125(2)$ & $51(1)$ \\
\hline$C(6)$ & 10998(4) & $9950(5)$ & $4320(2)$ & $54(1)$ \\
\hline$C(7)$ & 11655(4) & $10451(5)$ & $3895(2)$ & $56(1)$ \\
\hline $\mathrm{C}(8)$ & 11439(4) & $10125(5)$ & $3272(2)$ & $54(1)$ \\
\hline $\mathrm{C}(9)$ & $10478(4)$ & 9309(4) & $3045(2)$ & $45(1)$ \\
\hline $\mathrm{C}(10)$ & $10206(4)$ & 8942(4) & $2388(2)$ & $47(1)$ \\
\hline $\mathrm{C}(11)$ & 11167(4) & 9307(4) & 1999(2) & $47(1)$ \\
\hline $\mathrm{C}(12)$ & $12406(4)$ & $8743(5)$ & $2158(2)$ & $55(1)$ \\
\hline $\mathrm{C}(13)$ & $13478(4)$ & $9404(6)$ & 2034(3) & $65(2)$ \\
\hline $\mathrm{C}(14)$ & $13383(5)$ & $10442(6)$ & 1719(3) & $70(2)$ \\
\hline $\mathrm{C}(15)$ & $12153(5)$ & $11050(4)$ & $1547(2)$ & $56(1)$ \\
\hline $\mathrm{C}(16)$ & 12097(7) & 12132(6) & $1256(3)$ & $81(2)$ \\
\hline$C(17)$ & 10974(6) & $12755(5)$ & $1168(3)$ & $74(2)$ \\
\hline $\mathrm{C}(18)$ & $9987(6)$ & $12323(5)$ & $1376(3)$ & $66(2)$ \\
\hline $\mathrm{C}(19)$ & 10022(5) & $11210(5)$ & $1659(2)$ & $61(1)$ \\
\hline$C(20)$ & 11134(4) & $10514(4)$ & $1737(2)$ & $39(1)$ \\
\hline $\mathrm{C}(21)$ & 6893(4) & $8324(5)$ & $1188(2)$ & $56(1)$ \\
\hline $\mathrm{C}(22)$ & $5996(5)$ & $7602(6)$ & $1505(3)$ & $79(2)$ \\
\hline$C(23)$ & $6458(5)$ & $8267(7)$ & $469(3)$ & $86(2)$ \\
\hline $\mathrm{C}(24)$ & $6840(6)$ & $9646(5)$ & 1383(4) & $103(2)$ \\
\hline$C(25)$ & $8858(4)$ & $6337(4)$ & $1220(2)$ & $60(1)$ \\
\hline$C(26)$ & $7814(11)$ & $5481(8)$ & $1290(8)$ & $85(6)$ \\
\hline$C(26 \mathrm{~A})$ & 8387(13) & 5499(9) & 1804(7) & $63(4)$ \\
\hline $\mathrm{C}(27)$ & $10175(12)$ & $6041(14)$ & $1626(7)$ & $75(4)$ \\
\hline
\end{tabular}




\begin{tabular}{lclrl}
$\mathrm{C}(27 \mathrm{~A})$ & $10293(12)$ & $6078(15)$ & $1321(9)$ & $73(5)$ \\
$\mathrm{C}(28)$ & $9051(12)$ & $6258(9)$ & $466(4)$ & $65(4)$ \\
$\mathrm{C}(28 \mathrm{~A})$ & $8078(15)$ & $5931(13)$ & $570(6)$ & $96(7)$ \\
$\mathrm{C}(29)$ & $2170(5)$ & $8195(5)$ & $-53(2)$ & $56(1)$ \\
$\mathrm{C}(30)$ & $1365(5)$ & $9108(5)$ & $111(2)$ & $64(1)$ \\
\hline
\end{tabular}


Table 3. Bond lengths $[\AA]$ and angles $\left[{ }^{\circ}\right]$ for $\left(\mathrm{P}_{1} \mathrm{O}_{2} \mathrm{C}_{28} \mathrm{H}_{30}\right) \cdot\left(\mathrm{N}_{1} \mathrm{C}_{2} \mathrm{H}_{3}\right)$ (compound 2).

\begin{tabular}{|c|c|}
\hline $\mathrm{P}(1)-\mathrm{O}(1)$ & $1.482(3)$ \\
\hline $\mathrm{P}(1)-\mathrm{C}(25)$ & $1.833(5)$ \\
\hline $\mathrm{P}(1)-\mathrm{C}(1)$ & $1.840(4)$ \\
\hline $\mathrm{P}(1)-\mathrm{C}(21)$ & $1.879(5)$ \\
\hline $\mathrm{N}(1)-\mathrm{C}(29)$ & $1.136(6)$ \\
\hline $\mathrm{O}(2)-\mathrm{C}(11)$ & $1.437(5)$ \\
\hline $\mathrm{O}(2)-\mathrm{C}(12)$ & $1.441(5)$ \\
\hline $\mathrm{C}(1)-\mathrm{C}(10)$ & $1.395(6)$ \\
\hline $\mathrm{C}(1)-\mathrm{C}(2)$ & $1.403(6)$ \\
\hline$C(2)-C(3)$ & $1.359(6)$ \\
\hline $\mathrm{C}(3)-\mathrm{C}(4)$ & $1.400(7)$ \\
\hline $\mathrm{C}(4)-\mathrm{C}(5)$ & $1.417(6)$ \\
\hline $\mathrm{C}(4)-\mathrm{C}(9)$ & $1.420(6)$ \\
\hline $\mathrm{C}(5)-\mathrm{C}(6)$ & $1.345(7)$ \\
\hline $\mathrm{C}(6)-\mathrm{C}(7)$ & $1.386(7)$ \\
\hline $\mathrm{C}(7)-\mathrm{C}(8)$ & $1.369(6)$ \\
\hline $\mathrm{C}(8)-\mathrm{C}(9)$ & $1.415(6)$ \\
\hline$C(9)-C(10)$ & $1.450(6)$ \\
\hline $\mathrm{C}(10)-\mathrm{C}(11)$ & $1.520(6)$ \\
\hline$C(11)-C(20)$ & $1.463(6)$ \\
\hline $\mathrm{C}(11)-\mathrm{C}(12)$ & $1.484(6)$ \\
\hline$C(12)-C(13)$ & $1.458(7)$ \\
\hline$C(13)-C(14)$ & $1.341(8)$ \\
\hline$C(14)-C(15)$ & $1.499(8)$ \\
\hline$C(15)-C(16)$ & $1.361(8)$ \\
\hline$C(15)-C(20)$ & $1.397(6)$ \\
\hline$C(16)-C(17)$ & 1.401(9) \\
\hline $\mathrm{C}(17)-\mathrm{C}(18)$ & $1.339(8)$ \\
\hline $\mathrm{C}(18)-\mathrm{C}(19)$ & $1.384(7)$ \\
\hline$C(19)-C(20)$ & $1.434(7)$ \\
\hline$C(21)-C(22)$ & $1.530(8)$ \\
\hline $\mathrm{C}(21)-\mathrm{C}(23)$ & $1.535(8)$ \\
\hline$C(21)-C(24)$ & $1.543(8)$ \\
\hline$C(25)-C(27 A)$ & $1.580(13)$ \\
\hline$C(25)-C(28 A)$ & $1.569(11)$ \\
\hline$C(25)-C(26)$ & $1.524(10)$ \\
\hline
\end{tabular}




\begin{tabular}{|c|c|}
\hline $\mathrm{C}(25)-\mathrm{C}(27)$ & $1.583(12)$ \\
\hline$C(25)-C(28)$ & $1.680(10)$ \\
\hline $\mathrm{C}(25)-\mathrm{C}(26 \mathrm{~A})$ & $1.722(12)$ \\
\hline$C(26)-C(26 A)$ & $1.168(15)$ \\
\hline $\mathrm{C}(27)-\mathrm{C}(27 \mathrm{~A})$ & $0.693(15)$ \\
\hline $\mathrm{C}(28)-\mathrm{C}(28 \mathrm{~A})$ & $1.192(16)$ \\
\hline C(29)-C(30) & $1.439(7)$ \\
\hline $\mathrm{O}(1)-\mathrm{P}(1)-\mathrm{C}(25)$ & $110.7(2)$ \\
\hline $\mathrm{O}(1)-\mathrm{P}(1)-\mathrm{C}(1)$ & $112.25(18)$ \\
\hline $\mathrm{C}(25)-\mathrm{P}(1)-\mathrm{C}(1)$ & $106.6(2)$ \\
\hline $\mathrm{O}(1)-\mathrm{P}(1)-\mathrm{C}(21)$ & $107.8(2)$ \\
\hline $\mathrm{C}(25)-\mathrm{P}(1)-\mathrm{C}(21)$ & $112.9(2)$ \\
\hline $\mathrm{C}(1)-\mathrm{P}(1)-\mathrm{C}(21)$ & $106.6(2)$ \\
\hline $\mathrm{C}(11)-\mathrm{O}(2)-\mathrm{C}(12)$ & $62.1(3)$ \\
\hline $\mathrm{C}(10)-\mathrm{C}(1)-\mathrm{C}(2)$ & $118.9(4)$ \\
\hline $\mathrm{C}(10)-\mathrm{C}(1)-\mathrm{P}(1)$ & $122.6(3)$ \\
\hline $\mathrm{C}(2)-\mathrm{C}(1)-\mathrm{P}(1)$ & $118.6(3)$ \\
\hline $\mathrm{C}(3)-\mathrm{C}(2)-\mathrm{C}(1)$ & $122.5(4)$ \\
\hline $\mathrm{C}(2)-\mathrm{C}(3)-\mathrm{C}(4)$ & $120.6(4)$ \\
\hline$C(3)-C(4)-C(5)$ & $122.3(4)$ \\
\hline $\mathrm{C}(3)-\mathrm{C}(4)-\mathrm{C}(9)$ & $118.8(4)$ \\
\hline $\mathrm{C}(5)-\mathrm{C}(4)-\mathrm{C}(9)$ & $118.8(4)$ \\
\hline$C(6)-C(5)-C(4)$ & $120.9(4)$ \\
\hline$C(5)-C(6)-C(7)$ & $120.3(4)$ \\
\hline $\mathrm{C}(8)-\mathrm{C}(7)-\mathrm{C}(6)$ & $121.5(5)$ \\
\hline $\mathrm{C}(7)-\mathrm{C}(8)-\mathrm{C}(9)$ & 119.7(4) \\
\hline$C(8)-C(9)-C(4)$ & $118.6(4)$ \\
\hline$C(8)-C(9)-C(10)$ & $121.8(4)$ \\
\hline $\mathrm{C}(4)-\mathrm{C}(9)-\mathrm{C}(10)$ & 119.6(4) \\
\hline $\mathrm{C}(1)-\mathrm{C}(10)-\mathrm{C}(9)$ & $118.8(4)$ \\
\hline $\mathrm{C}(1)-\mathrm{C}(10)-\mathrm{C}(11)$ & $126.1(4)$ \\
\hline $\mathrm{C}(9)-\mathrm{C}(10)-\mathrm{C}(11)$ & $115.0(4)$ \\
\hline $\mathrm{O}(2)-\mathrm{C}(11)-\mathrm{C}(20)$ & $114.2(3)$ \\
\hline $\mathrm{O}(2)-\mathrm{C}(11)-\mathrm{C}(12)$ & $59.1(3)$ \\
\hline$C(20)-C(11)-C(12)$ & $116.1(4)$ \\
\hline $\mathrm{O}(2)-\mathrm{C}(11)-\mathrm{C}(10)$ & $116.5(4)$ \\
\hline$C(20)-C(11)-C(10)$ & $119.3(4)$ \\
\hline
\end{tabular}




\begin{tabular}{|c|c|}
\hline $\mathrm{C}(12)-\mathrm{C}(11)-\mathrm{C}(10)$ & $117.4(4)$ \\
\hline $\mathrm{O}(2)-\mathrm{C}(12)-\mathrm{C}(13)$ & $114.3(4)$ \\
\hline $\mathrm{O}(2)-\mathrm{C}(12)-\mathrm{C}(11)$ & $58.8(3)$ \\
\hline $\mathrm{C}(13)-\mathrm{C}(12)-\mathrm{C}(11)$ & $118.7(5)$ \\
\hline $\mathrm{C}(14)-\mathrm{C}(13)-\mathrm{C}(12)$ & $122.9(5)$ \\
\hline $\mathrm{C}(13)-\mathrm{C}(14)-\mathrm{C}(15)$ & $120.1(5)$ \\
\hline$C(16)-C(15)-C(20)$ & $123.1(5)$ \\
\hline $\mathrm{C}(16)-\mathrm{C}(15)-\mathrm{C}(14)$ & $118.7(5)$ \\
\hline $\mathrm{C}(20)-\mathrm{C}(15)-\mathrm{C}(14)$ & $118.0(5)$ \\
\hline$C(15)-C(16)-C(17)$ & $118.1(6)$ \\
\hline $\mathrm{C}(18)-\mathrm{C}(17)-\mathrm{C}(16)$ & $121.5(6)$ \\
\hline $\mathrm{C}(17)-\mathrm{C}(18)-\mathrm{C}(19)$ & $120.9(6)$ \\
\hline $\mathrm{C}(18)-\mathrm{C}(19)-\mathrm{C}(20)$ & $119.8(5)$ \\
\hline$C(15)-C(20)-C(19)$ & $116.4(4)$ \\
\hline$C(15)-C(20)-C(11)$ & $123.0(4)$ \\
\hline$C(19)-C(20)-C(11)$ & $120.6(4)$ \\
\hline $\mathrm{C}(22)-\mathrm{C}(21)-\mathrm{C}(23)$ & $108.7(4)$ \\
\hline$C(22)-C(21)-C(24)$ & $109.0(5)$ \\
\hline$C(23)-C(21)-C(24)$ & $107.1(5)$ \\
\hline $\mathrm{C}(22)-\mathrm{C}(21)-\mathrm{P}(1)$ & $119.6(4)$ \\
\hline $\mathrm{C}(23)-\mathrm{C}(21)-\mathrm{P}(1)$ & $106.4(3)$ \\
\hline $\mathrm{C}(24)-\mathrm{C}(21)-\mathrm{P}(1)$ & $105.3(4)$ \\
\hline $\mathrm{C}(27 \mathrm{~A})-\mathrm{C}(25)-\mathrm{C}(28 \mathrm{~A})$ & $116.0(8)$ \\
\hline$C(27 A)-C(25)-C(26)$ & $128.5(9)$ \\
\hline $\mathrm{C}(28 \mathrm{~A})-\mathrm{C}(25)-\mathrm{C}(26)$ & $66.9(8)$ \\
\hline $\mathrm{C}(27 \mathrm{~A})-\mathrm{C}(25)-\mathrm{C}(27)$ & $25.3(6)$ \\
\hline $\mathrm{C}(28 \mathrm{~A})-\mathrm{C}(25)-\mathrm{C}(27)$ & $136.6(7)$ \\
\hline$C(26)-C(25)-C(27)$ & $116.3(8)$ \\
\hline $\mathrm{C}(27 \mathrm{~A})-\mathrm{C}(25)-\mathrm{C}(28)$ & $79.8(7)$ \\
\hline $\mathrm{C}(28 \mathrm{~A})-\mathrm{C}(25)-\mathrm{C}(28)$ & $42.9(6)$ \\
\hline$C(26)-C(25)-C(28)$ & $107.1(6)$ \\
\hline $\mathrm{C}(27)-\mathrm{C}(25)-\mathrm{C}(28)$ & $104.9(6)$ \\
\hline $\mathrm{C}(27 \mathrm{~A})-\mathrm{C}(25)-\mathrm{C}(26 \mathrm{~A})$ & $103.1(8)$ \\
\hline $\mathrm{C}(28 \mathrm{~A})-\mathrm{C}(25)-\mathrm{C}(26 \mathrm{~A})$ & $107.7(7)$ \\
\hline$C(26)-C(25)-C(26 A)$ & $41.6(6)$ \\
\hline$C(27)-C(25)-C(26 A)$ & $81.4(7)$ \\
\hline $\mathrm{C}(28)-\mathrm{C}(25)-\mathrm{C}(26 \mathrm{~A})$ & $141.8(6)$ \\
\hline $\mathrm{C}(27 \mathrm{~A})-\mathrm{C}(25)-\mathrm{P}(1)$ & $110.3(7)$ \\
\hline
\end{tabular}




$\begin{array}{lc}\mathrm{C}(28 \mathrm{~A})-\mathrm{C}(25)-\mathrm{P}(1) & 109.3(6) \\ \mathrm{C}(26)-\mathrm{C}(25)-\mathrm{P}(1) & 116.7(5) \\ \mathrm{C}(27)-\mathrm{C}(25)-\mathrm{P}(1) & 106.5(6) \\ \mathrm{C}(28)-\mathrm{C}(25)-\mathrm{P}(1) & 104.0(4) \\ \mathrm{C}(26 \mathrm{~A})-\mathrm{C}(25)-\mathrm{P}(1) & 110.2(5) \\ \mathrm{C}(26 \mathrm{~A})-\mathrm{C}(26)-\mathrm{C}(25) & 78.3(8) \\ \mathrm{C}(26)-\mathrm{C}(26 \mathrm{~A})-\mathrm{C}(25) & 60.1(7) \\ \mathrm{C}(27 \mathrm{~A})-\mathrm{C}(27)-\mathrm{C}(25) & 77.1(17) \\ \mathrm{C}(27)-\mathrm{C}(27 \mathrm{~A})-\mathrm{C}(25) & 77.6(17) \\ \mathrm{C}(28 \mathrm{~A})-\mathrm{C}(28)-\mathrm{C}(25) & 63.6(7) \\ \mathrm{C}(28)-\mathrm{C}(28 \mathrm{~A})-\mathrm{C}(25) & 73.6(8) \\ \mathrm{N}(1)-\mathrm{C}(29)-\mathrm{C}(30) & 179.7(8)\end{array}$

Symmetry transformations used to generate equivalent atoms: 
Table 4. Anisotropic displacement parameters $\left(\AA^{2} \times 10^{3}\right)$ for $\left(\mathrm{P}_{1} \mathrm{O}_{2} \mathrm{C}_{28} \mathrm{H}_{30}\right) \cdot\left(\mathrm{N}_{1} \mathrm{C}_{2} \mathrm{H}_{3}\right)$ (compound 2). The anisotropic displacement factor exponent takes the form: $-2 \pi^{2}\left[h^{2} a^{* 2} U^{11}+\right.$ $\ldots+2 \mathrm{hk} \mathrm{a}^{*} \mathrm{~b}^{*} \mathrm{U}^{12}$ ]

\begin{tabular}{|c|c|c|c|c|c|c|}
\hline & $\mathrm{U}^{11}$ & $\mathrm{U}^{22}$ & $\mathrm{U}^{33}$ & $\mathrm{U}^{23}$ & $\mathrm{U}^{13}$ & $\mathrm{U}^{12}$ \\
\hline $\mathrm{P}(1)$ & $38(1)$ & $42(1)$ & $43(1)$ & $-10(1)$ & $4(1)$ & $-3(1)$ \\
\hline $\mathrm{N}(1)$ & $95(4)$ & $99(4)$ & 74(3) & 6(3) & 21(3) & $47(3)$ \\
\hline $\mathrm{O}(1)$ & $43(2)$ & $51(2)$ & $39(2)$ & $-5(1)$ & $4(1)$ & 1(1) \\
\hline $\mathrm{O}(2)$ & $54(2)$ & $62(2)$ & $47(2)$ & $-7(2)$ & $13(2)$ & $8(2)$ \\
\hline $\mathrm{C}(1)$ & $48(3)$ & $43(3)$ & $44(3)$ & $-4(2)$ & $8(2)$ & $-8(2)$ \\
\hline$C(2)$ & $46(3)$ & $46(3)$ & $48(3)$ & $1(2)$ & $13(2)$ & $-5(2)$ \\
\hline$C(3)$ & $50(3)$ & 48(3) & $52(3)$ & $10(2)$ & $22(2)$ & $2(2)$ \\
\hline C(4) & $44(2)$ & 48(3) & $37(2)$ & $4(2)$ & $13(2)$ & $16(2)$ \\
\hline$C(5)$ & $51(3)$ & $69(3)$ & $37(2)$ & $6(2)$ & $15(2)$ & 19(3) \\
\hline$C(6)$ & $54(3)$ & 79(4) & $29(2)$ & $-8(2)$ & $6(2)$ & 19(3) \\
\hline$C(7)$ & $46(3)$ & 76(4) & $42(3)$ & $-15(2)$ & $0(2)$ & $2(2)$ \\
\hline $\mathrm{C}(8)$ & $50(3)$ & 73(3) & $38(3)$ & $-8(2)$ & $8(2)$ & $-6(2)$ \\
\hline $\mathrm{C}(9)$ & $49(3)$ & $51(3)$ & $34(2)$ & $-2(2)$ & $8(2)$ & $-3(2)$ \\
\hline $\mathrm{C}(10)$ & $53(3)$ & $51(3)$ & $38(2)$ & $3(2)$ & $8(2)$ & $-8(2)$ \\
\hline $\mathrm{C}(11)$ & $51(3)$ & $52(3)$ & $38(2)$ & $-7(2)$ & $7(2)$ & $5(2)$ \\
\hline $\mathrm{C}(12)$ & $39(3)$ & $81(4)$ & $46(3)$ & $-18(3)$ & $10(2)$ & 1(2) \\
\hline$C(13)$ & $38(3)$ & $88(4)$ & 71(4) & $-17(3)$ & $17(2)$ & $7(3)$ \\
\hline$C(14)$ & $52(3)$ & $88(5)$ & 74(4) & $-21(3)$ & 22(3) & $-16(3)$ \\
\hline $\mathrm{C}(15)$ & 74(4) & $51(3)$ & $45(3)$ & $-11(2)$ & $17(2)$ & $-22(3)$ \\
\hline $\mathrm{C}(16)$ & $115(5)$ & 72(4) & 64(4) & $-15(3)$ & $36(4)$ & $-39(4)$ \\
\hline $\mathrm{C}(17)$ & $96(5)$ & $51(3)$ & 74(4) & $-11(3)$ & $15(3)$ & $-6(3)$ \\
\hline $\mathrm{C}(18)$ & $81(4)$ & $40(3)$ & 73(4) & 7(3) & $5(3)$ & $5(3)$ \\
\hline $\mathrm{C}(19)$ & $70(3)$ & $52(3)$ & $55(3)$ & $-5(2)$ & $-1(2)$ & $-5(3)$ \\
\hline$C(20)$ & $40(2)$ & 44(3) & $34(2)$ & $-7(2)$ & $9(2)$ & $-10(2)$ \\
\hline $\mathrm{C}(21)$ & $40(3)$ & 59(3) & $67(3)$ & $-15(3)$ & $5(2)$ & $4(2)$ \\
\hline $\mathrm{C}(22)$ & $37(3)$ & $99(5)$ & $105(5)$ & $-14(4)$ & 21(3) & 2(3) \\
\hline $\mathrm{C}(23)$ & $49(3)$ & $130(6)$ & $70(4)$ & $0(4)$ & $-11(3)$ & $13(3)$ \\
\hline $\mathrm{C}(24)$ & 68(4) & $58(4)$ & $175(7)$ & $-29(4)$ & 2(4) & 21(3) \\
\hline$C(25)$ & $54(3)$ & 49(3) & $77(3)$ & $-14(3)$ & $9(3)$ & $8(2)$ \\
\hline$C(26)$ & $95(9)$ & $40(6)$ & $135(16)$ & $-25(7)$ & $62(10)$ & $-23(6)$ \\
\hline$C(26 \mathrm{~A})$ & 78(9) & $39(7)$ & $72(10)$ & $-9(6)$ & $18(8)$ & $-3(6)$ \\
\hline $\mathrm{C}(27)$ & $77(8)$ & $80(9)$ & 74(9) & $-2(8)$ & $28(7)$ & $40(7)$ \\
\hline
\end{tabular}




\begin{tabular}{lcccccc}
$\mathrm{C}(27 \mathrm{~A})$ & $61(9)$ & $77(10)$ & $86(13)$ & $-33(10)$ & $25(8)$ & $11(7)$ \\
$\mathrm{C}(28)$ & $83(10)$ & $60(6)$ & $56(6)$ & $-24(5)$ & $21(5)$ & $-9(6)$ \\
$\mathrm{C}(28 \mathrm{~A})$ & $81(12)$ & $96(11)$ & $108(12)$ & $-74(10)$ & $7(9)$ & $-7(9)$ \\
$\mathrm{C}(29)$ & $58(3)$ & $68(3)$ & $42(3)$ & $9(2)$ & $11(2)$ & $20(3)$ \\
$\mathrm{C}(30)$ & $80(4)$ & $65(3)$ & $50(3)$ & $2(3)$ & $24(3)$ & $19(3)$ \\
\hline
\end{tabular}


Table 5. Hydrogen coordinates ( $\left.\mathrm{x} 10^{4}\right)$ and isotropic displacement parameters $\left(\AA^{2} \times 10^{3}\right)$ for $\left(\mathrm{P}_{1} \mathrm{O}_{2} \mathrm{C}_{28} \mathrm{H}_{30}\right)^{\prime} \cdot\left(\mathrm{N}_{1} \mathrm{C}_{2} \mathrm{H}_{3}\right)$ (compound 2).

\begin{tabular}{|c|c|c|c|c|}
\hline & $\mathrm{x}$ & $\mathrm{y}$ & $\mathrm{z}$ & $\mathrm{U}(\mathrm{eq})$ \\
\hline $\mathrm{H}(2)$ & 7856 & 7229 & 2493 & 56 \\
\hline $\mathrm{H}(3)$ & 8421 & 7616 & 3549 & 58 \\
\hline $\mathrm{H}(5)$ & 9679 & 8786 & 4423 & 62 \\
\hline $\mathrm{H}(6)$ & 11179 & 10179 & 4752 & 65 \\
\hline $\mathrm{H}(7)$ & 12270 & 11033 & 4039 & 67 \\
\hline $\mathrm{H}(8)$ & 11932 & 10445 & 2993 & 64 \\
\hline $\mathrm{H}(13)$ & 14278 & 9084 & 2182 & 78 \\
\hline $\mathrm{H}(14)$ & 14096 & 10793 & 1604 & 84 \\
\hline $\mathrm{H}(16)$ & 12799 & 12455 & 1117 & 97 \\
\hline $\mathrm{H}(17)$ & 10911 & 13501 & 955 & 88 \\
\hline $\mathrm{H}(18)$ & 9253 & 12787 & 1330 & 79 \\
\hline $\mathrm{H}(19)$ & 9313 & 10909 & 1800 & 73 \\
\hline $\mathrm{H}(22 \mathrm{~A})$ & 5144 & 7785 & 1303 & 119 \\
\hline $\mathrm{H}(22 \mathrm{~B})$ & 6116 & 7810 & 1954 & 119 \\
\hline $\mathrm{H}(22 \mathrm{C})$ & 6153 & 6748 & 1461 & 119 \\
\hline $\mathrm{H}(23 \mathrm{~A})$ & 6321 & 7432 & 338 & 128 \\
\hline $\mathrm{H}(23 \mathrm{~B})$ & 7092 & 8617 & 259 & 128 \\
\hline $\mathrm{H}(23 \mathrm{C})$ & 5685 & 8714 & 352 & 128 \\
\hline $\mathrm{H}(24 \mathrm{~A})$ & 7414 & 10114 & 1182 & 154 \\
\hline $\mathrm{H}(24 \mathrm{~B})$ & 7076 & 9713 & 1843 & 154 \\
\hline $\mathrm{H}(24 \mathrm{C})$ & 5998 & 9950 & 1248 & 154 \\
\hline $\mathrm{H}(30 \mathrm{~A})$ & 1057 & 9598 & -262 & 95 \\
\hline $\mathrm{H}(30 \mathrm{~B})$ & 1825 & 9613 & 445 & 95 \\
\hline $\mathrm{H}(30 \mathrm{C})$ & 666 & 8736 & 261 & 95 \\
\hline
\end{tabular}




\section{X-ray Data of Compound 5}

X-ray diffraction data from a crystal of $\mathbf{5}$ obtained by slow crystallization from ethanol at room temperature were collected on a SMART APEX CCD diffractometer with graphite monochromated Mo-Ka radiation $(\lambda=0.71073 \AA)$ at $153 \mathrm{~K}$. The cell parameters for 5 were obtained from the least-squares refinement of the spots (from 60 collected frames) using the SMART program. A hemisphere of the crystal data was collected up to a resolution of $0.75 \AA$, the intensity data were processed using the Saint Plus program. All calculations for structure determination were carried out using the SHELXTL package (version 5.1) [1]. Initial atomic positions were located by direct methods using XS, and the structure was refined by least-squares methods using SHELX with 4822 independent reflections and within the range of theta $1.70-25.68^{\circ}$ (completeness 97.8\%). Absorption corrections were applied by using SADABS [2]. Calculated hydrogen positions were input and refined in a riding manner along with the attached carbons. A summary of the refinement details and the resulting factors are given in Table 6-10.

Table 6. Crystal data and structure refinement for $\left(\mathrm{C}_{28} \mathrm{H}_{31} \mathrm{O}_{2} \mathrm{P}\right) \cdot\left(\mathrm{O}_{1} \mathrm{C}_{2} \mathrm{H}_{6}\right)$ (compound 5).

$\begin{array}{ll}\text { Empirical formula } & \mathrm{C}_{30} \mathrm{H}_{37} \mathrm{O}_{3} \mathrm{P} \\ \text { Formula weight } & 476.57 \\ \text { Temperature } & 153(2) \mathrm{K} \\ \text { Wavelength } & 0.71073 \AA \\ \text { Crystal system } & \text { Triclinic } \\ \text { Space group } & \mathrm{P}-1 \\ \text { Unit cell dimensions } & \mathrm{a}=10.675(2) \AA \\ & \mathrm{b}=11.455(3) \AA \\ & \mathrm{c}=12.152(3) \AA \\ \text { Volume } & 1299.3(5) \AA^{3} \\ \text { Z } & 2 \\ \text { Density (calculated) } & 1.218 \mathrm{Mg} / \mathrm{m}^{3} \\ \text { Absorption coefficient } & 0.135 \mathrm{~mm}^{-1} \\ \text { F(000) } & 512 \\ \text { Crystal size } & 0.54 \times 0.22 \times 0.02 \mathrm{~mm}^{3} \\ \text { Theta range for data collection } & 1.70 \text { to } 25.68^{\circ} .\end{array}$


Index ranges

Reflections collected

Independent reflections

Completeness to theta $=25.68^{\circ}$

Refinement method

Data / restraints / parameters

Goodness-of-fit on $\mathrm{F}^{2}$

Final $\mathrm{R}$ indices [I $>2 \operatorname{sigma}(\mathrm{I})]$

$\mathrm{R}$ indices (all data)

Largest diff. peak and hole

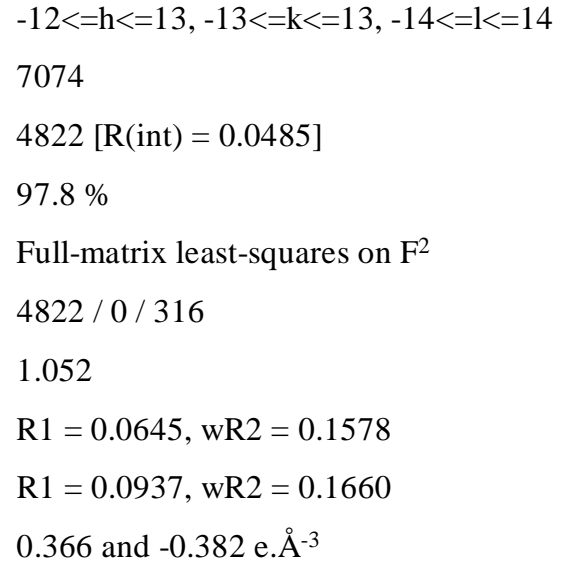

Table 7. Atomic coordinates and equivalent isotropic displacement parameters $\left(\AA^{2} \times 10^{3}\right)$ for $\left(\mathrm{C}_{28}\right.$ $\left.\mathrm{H}_{31} \mathrm{O}_{2} \mathrm{P}\right)^{\prime}\left(\mathrm{O}_{1} \mathrm{C}_{2} \mathrm{H}_{6}\right)$. U(eq) is defined as one third of the trace of the orthogonalized $\mathrm{U}^{\mathrm{ij}}$ tensor.

\begin{tabular}{|c|c|c|c|c|}
\hline & $\mathrm{x}$ & $\mathrm{y}$ & $\mathrm{z}$ & $\mathrm{U}(\mathrm{eq})$ \\
\hline $\mathrm{P}(1)$ & $8855(1)$ & $7553(1)$ & $7361(1)$ & $25(1)$ \\
\hline $\mathrm{O}(1)$ & $7505(2)$ & $6316(2)$ & $6924(2)$ & $29(1)$ \\
\hline $\mathrm{O}(2)$ & $6214(3)$ & $7351(2)$ & $4958(2)$ & $36(1)$ \\
\hline $\mathrm{O}(3)$ & $5264(3)$ & $4052(2)$ & $7027(2)$ & $38(1)$ \\
\hline$C(1)$ & $8639(4)$ & $9040(3)$ & $7316(3)$ & $26(1)$ \\
\hline$C(2)$ & $9917(4)$ & $10283(3)$ & $7559(3)$ & $29(1)$ \\
\hline$C(3)$ & $9904(4)$ & $11452(3)$ & $7505(3)$ & $30(1)$ \\
\hline$C(4)$ & $8624(4)$ & 11481(3) & $7162(3)$ & $29(1)$ \\
\hline$C(5)$ & $8592(4)$ & $12682(3)$ & $7059(3)$ & $37(1)$ \\
\hline$C(6)$ & $7362(4)$ & 12691(4) & $6663(3)$ & $44(1)$ \\
\hline$C(7)$ & $6095(4)$ & $11484(4)$ & $6368(3)$ & $41(1)$ \\
\hline $\mathrm{C}(8)$ & $6081(4)$ & $10306(4)$ & $6490(3)$ & $36(1)$ \\
\hline $\mathrm{C}(9)$ & $7349(4)$ & $10267(3)$ & $6890(3)$ & $27(1)$ \\
\hline$C(10)$ & $7369(4)$ & $9042(3)$ & $7002(3)$ & $27(1)$ \\
\hline $\mathrm{C}(11)$ & $5941(3)$ & $7816(3)$ & $6809(3)$ & $27(1)$ \\
\hline$C(12)$ & $5080(4)$ & $7532(3)$ & $7671(3)$ & $29(1)$ \\
\hline$C(13)$ & $5491(4)$ & 8399(4) & $8706(3)$ & $34(1)$ \\
\hline $\mathrm{C}(14)$ & $4641(4)$ & $8118(4)$ & $9521(3)$ & $41(1)$ \\
\hline$C(15)$ & $3348(4)$ & $6935(4)$ & $9360(3)$ & $45(1)$ \\
\hline$C(16)$ & $2925(4)$ & $6093(4)$ & $8379(3)$ & $39(1)$ \\
\hline $\mathrm{C}(17)$ & $3732(4)$ & $6374(3)$ & $7503(3)$ & $31(1)$ \\
\hline $\mathrm{C}(18)$ & $3267(4)$ & $5554(4)$ & $6464(3)$ & $34(1)$ \\
\hline
\end{tabular}




\begin{tabular}{lrlll}
$\mathrm{C}(19)$ & $4065(4)$ & $5864(3)$ & $5621(3)$ & $31(1)$ \\
$\mathrm{C}(20)$ & $5412(4)$ & $7011(3)$ & $5795(3)$ & $28(1)$ \\
$\mathrm{C}(21)$ & $10197(4)$ & $7673(3)$ & $6447(3)$ & $30(1)$ \\
$\mathrm{C}(22)$ & $9943(4)$ & $6258(4)$ & $6070(3)$ & $39(1)$ \\
$\mathrm{C}(23)$ & $9853(5)$ & $8199(4)$ & $5398(3)$ & $48(1)$ \\
$\mathrm{C}(24)$ & $11783(4)$ & $8503(4)$ & $6928(4)$ & $48(1)$ \\
$\mathrm{C}(25)$ & $9379(4)$ & $7504(3)$ & $8851(3)$ & $30(1)$ \\
$\mathrm{C}(26)$ & $9506(4)$ & $6229(4)$ & $8907(3)$ & $41(1)$ \\
$\mathrm{C}(27)$ & $10744(4)$ & $8686(4)$ & $9462(3)$ & $41(1)$ \\
$\mathrm{C}(28)$ & $8117(4)$ & $7382(4)$ & $9428(3)$ & $37(1)$ \\
$\mathrm{C}(29)$ & $3745(5)$ & $2400(4)$ & $7985(4)$ & $57(1)$ \\
$\mathrm{C}(30)$ & $4849(5)$ & $3791(4)$ & $8073(3)$ & $48(1)$ \\
\hline
\end{tabular}

Table 8. Bond lengths $[\AA]$ and angles $\left[^{\circ}\right]$ for $\left(\mathrm{C}_{28} \mathrm{H}_{31} \mathrm{O}_{2} \mathrm{P}\right) \cdot\left(\mathrm{O}_{1} \mathrm{C}_{2} \mathrm{H}_{6}\right)$.

\begin{tabular}{ll}
\hline $\mathrm{P}(1)-\mathrm{O}(1)$ & $1.488(2)$ \\
$\mathrm{P}(1)-\mathrm{C}(1)$ & $1.826(3)$ \\
$\mathrm{P}(1)-\mathrm{C}(25)$ & $1.855(4)$ \\
$\mathrm{P}(1)-\mathrm{C}(21)$ & $1.862(4)$ \\
$\mathrm{O}(2)-\mathrm{C}(20)$ & $1.361(4)$ \\
$\mathrm{O}(3)-\mathrm{C}(30)$ & $1.398(5)$ \\
$\mathrm{C}(1)-\mathrm{C}(10)$ & $1.372(5)$ \\
$\mathrm{C}(1)-\mathrm{C}(2)$ & $1.428(5)$ \\
$\mathrm{C}(2)-\mathrm{C}(3)$ & $1.353(4)$ \\
$\mathrm{C}(3)-\mathrm{C}(4)$ & $1.406(5)$ \\
$\mathrm{C}(4)-\mathrm{C}(5)$ & $1.408(5)$ \\
$\mathrm{C}(4)-\mathrm{C}(9)$ & $1.411(5)$ \\
$\mathrm{C}(5)-\mathrm{C}(6)$ & $1.358(5)$ \\
$\mathrm{C}(6)-\mathrm{C}(7)$ & $1.404(5)$ \\
$\mathrm{C}(7)-\mathrm{C}(8)$ & $1.364(5)$ \\
$\mathrm{C}(8)-\mathrm{C}(9)$ & $1.414(5)$ \\
$\mathrm{C}(9)-\mathrm{C}(10)$ & $1.432(5)$ \\
$\mathrm{C}(10)-\mathrm{C}(11)$ & $1.503(5)$ \\
$\mathrm{C}(11)-\mathrm{C}(20)$ & $1.370(5)$ \\
$\mathrm{C}(11)-\mathrm{C}(12)$ & $1.423(5)$ \\
$\mathrm{C}(12)-\mathrm{C}(17)$ & $1.419(5)$ \\
$\mathrm{C}(12)-\mathrm{C}(13)$ & $1.426(5)$ \\
\end{tabular}




\begin{tabular}{|c|c|}
\hline C(13)-C(14) & $1.369(5)$ \\
\hline $\mathrm{C}(14)-\mathrm{C}(15)$ & $1.402(5)$ \\
\hline $\mathrm{C}(15)-\mathrm{C}(16)$ & $1.360(5)$ \\
\hline$C(16)-C(17)$ & $1.402(5)$ \\
\hline $\mathrm{C}(17)-\mathrm{C}(18)$ & $1.402(5)$ \\
\hline $\mathrm{C}(18)-\mathrm{C}(19)$ & $1.364(5)$ \\
\hline C(19)-C(20) & $1.413(5)$ \\
\hline $\mathrm{C}(21)-\mathrm{C}(24)$ & $1.531(5)$ \\
\hline $\mathrm{C}(21)-\mathrm{C}(22)$ & $1.534(5)$ \\
\hline $\mathrm{C}(21)-\mathrm{C}(23)$ & $1.539(5)$ \\
\hline$C(25)-C(27)$ & $1.520(5)$ \\
\hline$C(25)-C(28)$ & $1.535(5)$ \\
\hline $\mathrm{C}(25)-\mathrm{C}(26)$ & $1.535(5)$ \\
\hline C(29)-C(30) & $1.471(5)$ \\
\hline $\mathrm{O}(1)-\mathrm{P}(1)-\mathrm{C}(1)$ & $112.13(15)$ \\
\hline $\mathrm{O}(1)-\mathrm{P}(1)-\mathrm{C}(25)$ & $108.07(15)$ \\
\hline $\mathrm{C}(1)-\mathrm{P}(1)-\mathrm{C}(25)$ & $107.01(15)$ \\
\hline $\mathrm{O}(1)-\mathrm{P}(1)-\mathrm{C}(21)$ & $108.11(15)$ \\
\hline $\mathrm{C}(1)-\mathrm{P}(1)-\mathrm{C}(21)$ & $106.97(15)$ \\
\hline $\mathrm{C}(25)-\mathrm{P}(1)-\mathrm{C}(21)$ & $114.65(17)$ \\
\hline $\mathrm{C}(10)-\mathrm{C}(1)-\mathrm{C}(2)$ & $118.6(3)$ \\
\hline $\mathrm{C}(10)-\mathrm{C}(1)-\mathrm{P}(1)$ & $125.0(3)$ \\
\hline $\mathrm{C}(2)-\mathrm{C}(1)-\mathrm{P}(1)$ & $116.3(3)$ \\
\hline $\mathrm{C}(3)-\mathrm{C}(2)-\mathrm{C}(1)$ & $121.9(3)$ \\
\hline $\mathrm{C}(2)-\mathrm{C}(3)-\mathrm{C}(4)$ & $120.7(3)$ \\
\hline$C(3)-C(4)-C(5)$ & $121.7(3)$ \\
\hline $\mathrm{C}(3)-\mathrm{C}(4)-\mathrm{C}(9)$ & $118.6(3)$ \\
\hline$C(5)-C(4)-C(9)$ & $119.6(3)$ \\
\hline$C(6)-C(5)-C(4)$ & 121.1(4) \\
\hline$C(5)-C(6)-C(7)$ & $119.5(4)$ \\
\hline$C(8)-C(7)-C(6)$ & $120.8(4)$ \\
\hline $\mathrm{C}(7)-\mathrm{C}(8)-\mathrm{C}(9)$ & $120.8(3)$ \\
\hline $\mathrm{C}(8)-\mathrm{C}(9)-\mathrm{C}(4)$ & 118.1(3) \\
\hline C(8)-C(9)-C(10) & $121.9(3)$ \\
\hline$C(4)-C(9)-C(10)$ & $120.0(3)$ \\
\hline $\mathrm{C}(1)-\mathrm{C}(10)-\mathrm{C}(9)$ & $120.0(3)$ \\
\hline $\mathrm{C}(1)-\mathrm{C}(10)-\mathrm{C}(11)$ & $123.8(3)$ \\
\hline $\mathrm{C}(9)-\mathrm{C}(10)-\mathrm{C}(11)$ & 116.1(3) \\
\hline
\end{tabular}




$\begin{array}{ll}\mathrm{C}(20)-\mathrm{C}(11)-\mathrm{C}(12) & 119.2(3) \\ \mathrm{C}(20)-\mathrm{C}(11)-\mathrm{C}(10) & 121.2(3) \\ \mathrm{C}(12)-\mathrm{C}(11)-\mathrm{C}(10) & 119.4(3) \\ \mathrm{C}(11)-\mathrm{C}(12)-\mathrm{C}(17) & 120.0(3) \\ \mathrm{C}(11)-\mathrm{C}(12)-\mathrm{C}(13) & 122.4(3) \\ \mathrm{C}(17)-\mathrm{C}(12)-\mathrm{C}(13) & 117.5(3) \\ \mathrm{C}(14)-\mathrm{C}(13)-\mathrm{C}(12) & 121.6(4) \\ \mathrm{C}(13)-\mathrm{C}(14)-\mathrm{C}(15) & 119.9(4) \\ \mathrm{C}(16)-\mathrm{C}(15)-\mathrm{C}(14) & 119.7(4) \\ \mathrm{C}(15)-\mathrm{C}(16)-\mathrm{C}(17) & 122.2(4) \\ \mathrm{C}(18)-\mathrm{C}(17)-\mathrm{C}(16) & 122.7(3) \\ \mathrm{C}(18)-\mathrm{C}(17)-\mathrm{C}(12) & 118.4(3) \\ \mathrm{C}(16)-\mathrm{C}(17)-\mathrm{C}(12) & 118.9(4) \\ \mathrm{C}(19)-\mathrm{C}(18)-\mathrm{C}(17) & 121.4(3) \\ \mathrm{C}(18)-\mathrm{C}(19)-\mathrm{C}(20) & 120.0(3) \\ \mathrm{O}(2)-\mathrm{C}(20)-\mathrm{C}(11) & 118.2(3) \\ \mathrm{O}(2)-\mathrm{C}(20)-\mathrm{C}(19) & 121.0(3) \\ \mathrm{C}(11)-\mathrm{C}(20)-\mathrm{C}(19) & 120.8(3) \\ \mathrm{C}(24)-\mathrm{C}(21)-\mathrm{C}(22) & 106.8(3) \\ \mathrm{C}(24)-\mathrm{C}(21)-\mathrm{C}(23) & 109.7(3) \\ \mathrm{C}(22)-\mathrm{C}(21)-\mathrm{C}(23) & 107.2(3) \\ \mathrm{C}(24)-\mathrm{C}(21)-\mathrm{P}(1) & 118.5(3) \\ \mathrm{C}(22)-\mathrm{C}(21)-\mathrm{P}(1) & 107.6(2) \\ \mathrm{C}(23)-\mathrm{C}(21)-\mathrm{P}(1) & 106.5(2) \\ \mathrm{C}(27)-\mathrm{C}(25)-\mathrm{C}(28) & 109.4(3) \\ \mathrm{C}(27)-\mathrm{C}(25)-\mathrm{C}(26) & 109.3(3) \\ \mathrm{C}(28)-\mathrm{C}(25)-\mathrm{C}(26) & 108.2(3) \\ \mathrm{C}(27)-\mathrm{C}(25)-\mathrm{P}(1) & 116.6(3) \\ \mathrm{C}(28)-\mathrm{C}(25)-\mathrm{P}(1) & 105.3(2) \\ \mathrm{C}(26)-\mathrm{C}(25)-\mathrm{P}(1) & 107.6(2) \\ \mathrm{O}(3)-\mathrm{C}(30)-\mathrm{C}(29) & 109.7(3)\end{array}$

\section{Symmetry transformations used to generate equivalent atoms:}

Table 9. Anisotropic displacement parameters $\left(\AA^{2} \times 10^{3}\right)$ for $\left(\mathrm{C}_{28} \mathrm{H}_{31} \mathrm{O}_{2} \mathrm{P}\right) \cdot\left(\mathrm{O}_{1} \mathrm{C}_{2} \mathrm{H}_{6}\right)$. The anisotropic displacement factor exponent takes the form: $-2 \pi^{2}\left[h^{2} a^{* 2} U^{11}+\ldots+2 h k a^{*} b^{*} U^{12}\right]$

$\mathrm{U}^{11} \quad \mathrm{U}^{22} \quad \mathrm{U}^{33} \quad \mathrm{U}^{23} \quad \mathrm{U}^{13} \quad \mathrm{U}^{12}$




\begin{tabular}{|c|c|c|c|c|c|c|}
\hline $\mathrm{P}(1)$ & $22(1)$ & $22(1)$ & $30(1)$ & 1(1) & 2(1) & $10(1)$ \\
\hline $\mathrm{O}(1)$ & $24(1)$ & $24(1)$ & $34(1)$ & $-1(1)$ & 1(1) & $8(1)$ \\
\hline $\mathrm{O}(2)$ & $33(2)$ & $36(2)$ & $33(1)$ & $-1(1)$ & 1(1) & $12(1)$ \\
\hline $\mathrm{O}(3)$ & $33(2)$ & $27(1)$ & $40(2)$ & $4(1)$ & 1(1) & $5(1)$ \\
\hline $\mathrm{C}(1)$ & $28(2)$ & $23(2)$ & $25(2)$ & 2(1) & $4(2)$ & $12(2)$ \\
\hline $\mathrm{C}(2)$ & $24(2)$ & $27(2)$ & $33(2)$ & $0(2)$ & 2(2) & $11(2)$ \\
\hline $\mathrm{C}(3)$ & $25(2)$ & $22(2)$ & $35(2)$ & 2(2) & $3(2)$ & $6(2)$ \\
\hline $\mathrm{C}(4)$ & $31(2)$ & $28(2)$ & $29(2)$ & $3(2)$ & $6(2)$ & $14(2)$ \\
\hline $\mathrm{C}(5)$ & $40(2)$ & $24(2)$ & $45(2)$ & $4(2)$ & $10(2)$ & $15(2)$ \\
\hline $\mathrm{C}(6)$ & $52(3)$ & $38(2)$ & $51(3)$ & $9(2)$ & $10(2)$ & $30(2)$ \\
\hline$C(7)$ & $36(2)$ & $40(2)$ & $55(3)$ & $9(2)$ & $3(2)$ & $24(2)$ \\
\hline C(8) & $28(2)$ & $34(2)$ & $45(2)$ & 2(2) & 4(2) & $16(2)$ \\
\hline $\mathrm{C}(9)$ & $28(2)$ & $25(2)$ & $30(2)$ & $0(2)$ & $6(2)$ & $13(2)$ \\
\hline $\mathrm{C}(10)$ & $27(2)$ & $28(2)$ & $26(2)$ & $3(2)$ & $7(2)$ & $12(2)$ \\
\hline $\mathrm{C}(11)$ & $21(2)$ & $24(2)$ & $37(2)$ & $3(2)$ & $3(2)$ & $13(2)$ \\
\hline $\mathrm{C}(12)$ & $26(2)$ & $29(2)$ & $36(2)$ & $4(2)$ & 2(2) & $16(2)$ \\
\hline $\mathrm{C}(13)$ & $28(2)$ & $38(2)$ & $36(2)$ & $4(2)$ & $5(2)$ & $15(2)$ \\
\hline $\mathrm{C}(14)$ & $39(2)$ & $53(3)$ & $36(2)$ & $4(2)$ & $6(2)$ & $26(2)$ \\
\hline $\mathrm{C}(15)$ & $35(2)$ & $63(3)$ & $45(2)$ & $17(2)$ & 13(2) & $27(2)$ \\
\hline$C(16)$ & $26(2)$ & $42(2)$ & $49(2)$ & $12(2)$ & $7(2)$ & $16(2)$ \\
\hline $\mathrm{C}(17)$ & $24(2)$ & $31(2)$ & $39(2)$ & $11(2)$ & $6(2)$ & $13(2)$ \\
\hline $\mathrm{C}(18)$ & $24(2)$ & $30(2)$ & $46(2)$ & $7(2)$ & $-2(2)$ & $11(2)$ \\
\hline $\mathrm{C}(19)$ & $29(2)$ & $27(2)$ & $35(2)$ & $0(2)$ & $-1(2)$ & $13(2)$ \\
\hline $\mathrm{C}(20)$ & $21(2)$ & $32(2)$ & $34(2)$ & $4(2)$ & $3(2)$ & $15(2)$ \\
\hline $\mathrm{C}(21)$ & $28(2)$ & $26(2)$ & $38(2)$ & $3(2)$ & $6(2)$ & $14(2)$ \\
\hline $\mathrm{C}(22)$ & $37(2)$ & $42(2)$ & $44(2)$ & $0(2)$ & $8(2)$ & $23(2)$ \\
\hline $\mathrm{C}(23)$ & $69(3)$ & $56(3)$ & $38(2)$ & $16(2)$ & $23(2)$ & $40(2)$ \\
\hline C(24) & $36(2)$ & $42(2)$ & $63(3)$ & $-3(2)$ & $16(2)$ & $16(2)$ \\
\hline $\mathrm{C}(25)$ & $30(2)$ & $29(2)$ & $31(2)$ & $3(2)$ & $4(2)$ & $14(2)$ \\
\hline$C(26)$ & $47(3)$ & $41(2)$ & $39(2)$ & $12(2)$ & 6(2) & $24(2)$ \\
\hline $\mathrm{C}(27)$ & $36(2)$ & $45(2)$ & $36(2)$ & 1(2) & $-1(2)$ & $17(2)$ \\
\hline $\mathrm{C}(28)$ & $35(2)$ & $43(2)$ & $30(2)$ & $3(2)$ & $7(2)$ & $17(2)$ \\
\hline $\mathrm{C}(29)$ & $41(3)$ & $63(3)$ & $53(3)$ & $14(2)$ & $11(2)$ & $11(2)$ \\
\hline $\mathrm{C}(30)$ & $52(3)$ & $39(2)$ & $46(3)$ & $9(2)$ & $6(2)$ & $14(2)$ \\
\hline
\end{tabular}

Table 10. Hydrogen coordinates ( x 104) and isotropic displacement parameters $\left(\AA^{2} \times 10^{3}\right)$ for $\left(C_{28}\right.$ 
$\left.\mathrm{H}_{31} \mathrm{O}_{2} \mathrm{P}\right) \cdot\left(\mathrm{O}_{1} \mathrm{C}_{2} \mathrm{H}_{6}\right)$ (compound 5).

\begin{tabular}{|c|c|c|c|c|}
\hline & $\mathrm{x}$ & $\mathrm{y}$ & $\mathrm{z}$ & $\mathrm{U}(\mathrm{eq})$ \\
\hline $\mathrm{H}(2)$ & 5756 & 6819 & 4369 & 54 \\
\hline $\mathrm{H}(3 \mathrm{~A})$ & 6036 & 4762 & 7108 & 57 \\
\hline $\mathrm{H}(2 \mathrm{~A})$ & 10802 & 10288 & 7764 & 35 \\
\hline $\mathrm{H}(3)$ & 10770 & 12261 & 7701 & 36 \\
\hline $\mathrm{H}(5)$ & 9445 & 13500 & 7269 & 44 \\
\hline $\mathrm{H}(6)$ & 7358 & 13508 & 6588 & 52 \\
\hline $\mathrm{H}(7)$ & 5237 & 11488 & 6079 & 49 \\
\hline $\mathrm{H}(8)$ & 5209 & 9502 & 6305 & 43 \\
\hline $\mathrm{H}(13)$ & 6378 & 9192 & 8833 & 41 \\
\hline $\mathrm{H}(14)$ & 4926 & 8724 & 10194 & 49 \\
\hline $\mathrm{H}(15)$ & 2770 & 6722 & 9933 & 54 \\
\hline $\mathrm{H}(16)$ & 2054 & 5287 & 8284 & 46 \\
\hline $\mathrm{H}(18)$ & 2378 & 4765 & 6345 & 41 \\
\hline $\mathrm{H}(19)$ & 3715 & 5309 & 4916 & 37 \\
\hline $\mathrm{H}(22 \mathrm{~A})$ & 8945 & 5701 & 5724 & 59 \\
\hline $\mathrm{H}(22 \mathrm{~B})$ & 10150 & 5889 & 6720 & 59 \\
\hline $\mathrm{H}(22 \mathrm{C})$ & 10570 & 6280 & 5526 & 59 \\
\hline $\mathrm{H}(23 \mathrm{~A})$ & 10412 & 8120 & 4830 & 72 \\
\hline $\mathrm{H}(23 \mathrm{~B})$ & 10096 & 9134 & 5594 & 72 \\
\hline $\mathrm{H}(23 \mathrm{C})$ & 8834 & 7679 & 5101 & 72 \\
\hline $\mathrm{H}(24 \mathrm{~A})$ & 12359 & 8469 & 6359 & 72 \\
\hline $\mathrm{H}(24 \mathrm{~B})$ & 12005 & 8146 & 7581 & 72 \\
\hline $\mathrm{H}(24 \mathrm{C})$ & 11999 & 9426 & 7154 & 72 \\
\hline $\mathrm{H}(26 \mathrm{~A})$ & 10325 & 6298 & 8562 & 61 \\
\hline $\mathrm{H}(26 \mathrm{~B})$ & 8632 & 5466 & 8504 & 61 \\
\hline $\mathrm{H}(26 \mathrm{C})$ & 9642 & 6107 & 9690 & 61 \\
\hline $\mathrm{H}(27 \mathrm{~A})$ & 10664 & 9502 & 9429 & 61 \\
\hline $\mathrm{H}(27 \mathrm{~B})$ & 11554 & 8750 & 9107 & 61 \\
\hline $\mathrm{H}(27 \mathrm{C})$ & 10894 & 8570 & 10245 & 61 \\
\hline $\mathrm{H}(28 \mathrm{~A})$ & 8282 & 7256 & 10206 & 55 \\
\hline $\mathrm{H}(28 \mathrm{~B})$ & 7237 & 6619 & 9036 & 55 \\
\hline $\mathrm{H}(28 \mathrm{C})$ & 8026 & 8194 & 9411 & 55 \\
\hline $\mathrm{H}(29 \mathrm{~A})$ & 2945 & 2223 & 7408 & 85 \\
\hline
\end{tabular}




$\begin{array}{lllll}\mathrm{H}(29 \mathrm{~B}) & 3405 & 2239 & 8705 & 85 \\ \mathrm{H}(29 \mathrm{C}) & 4149 & 1811 & 7784 & 85 \\ \mathrm{H}(30 \mathrm{~A}) & 4470 & 4390 & 8339 & 58 \\ \mathrm{H}(30 \mathrm{~B}) & 5685 & 3959 & 8620 & 58\end{array}$

[1] Sheldrick, G. M. SHELXTL, version 6.14; Bruker Analytical X-ray System, Inc.:

Madison, WI, 1997 
Coordinates for Calculation of TS Barrier for Intramolecular Oxidation to Compound 7.

Phosphine (Compound 6)

Gas Phase Energy w ZP correction: -1839.076986 Hartrees

Coordinates:

\begin{tabular}{|c|c|c|c|}
\hline C1 & -2.901 & 3.813 & 2.074 \\
\hline $\mathrm{C} 2$ & -3.181 & 3.091 & 0.886 \\
\hline C3 & -1.68 & 4.49 & 2.188 \\
\hline $\mathrm{C} 4$ & -0.893 & 4.668 & 1.05 \\
\hline C5 & -1.268 & 4.068 & -0.158 \\
\hline C6 & -2.135 & 2.921 & -0.07 \\
\hline C7 & -4.505 & 2.658 & 0.66 \\
\hline C8 & -5.473 & 2.782 & 1.662 \\
\hline C9 & -5.147 & 3.377 & 2.883 \\
\hline C10 & -3.869 & 3.905 & 3.086 \\
\hline C17 & -2.707 & 0.549 & -0.598 \\
\hline C18 & -2.901 & -0.327 & -1.697 \\
\hline C19 & -1.918 & 1.734 & -0.78 \\
\hline C20 & -0.853 & 1.567 & -1.723 \\
\hline $\mathrm{C} 21$ & -1.061 & 0.726 & -2.834 \\
\hline C22 & -2.147 & -0.143 & -2.858 \\
\hline C23 & -3.765 & -1.431 & -1.58 \\
\hline C24 & -4.33 & -1.749 & -0.344 \\
\hline C25 & -4.004 & -0.997 & 0.784 \\
\hline C26 & -3.174 & 0.123 & 0.666 \\
\hline $\mathrm{H} 11$ & -1.453 & 4.977 & 3.009 \\
\hline $\mathrm{H} 12$ & -0.096 & 5.404 & 1.064 \\
\hline $\mathrm{H} 13$ & -4.776 & 2.214 & -0.291 \\
\hline $\mathrm{H} 14$ & -6.384 & 2.449 & 1.504 \\
\hline H15 & -5.898 & 3.467 & 3.658 \\
\hline H16 & -3.648 & 4.425 & 4.01 \\
\hline $\mathrm{H} 27$ & -3.933 & -2.016 & -2.352 \\
\hline $\mathrm{H} 28$ & -4.976 & -2.614 & -0.25 \\
\hline $\mathrm{H} 29$ & -4.353 & -1.257 & 1.666 \\
\hline $\mathrm{H} 30$ & -2.917 & 0.609 & 1.479 \\
\hline H31 & -2.279 & -0.797 & -3.71 \\
\hline H32 & -0.355 & 0.685 & -3.653 \\
\hline P33 & 0.679 & 2.079 & -1.402 \\
\hline C34 & 1.87 & 0.749 & 2.831 \\
\hline C35 & 2.04 & 2.081 & 2.44 \\
\hline C36 & 1.691 & 2.481 & 1.146 \\
\hline C37 & 1.169 & 1.549 & 0.24 \\
\hline C38 & 1.001 & 0.213 & 0.634 \\
\hline C39 & 1.351 & -0.184 & 1.92 \\
\hline
\end{tabular}




$\begin{array}{lrrr}\text { C45 } & 3.87 & 0.851 & -4.475 \\ \text { C46 } & 3.511 & 2.195 & -4.332 \\ \text { C47 } & 2.539 & 2.564 & -3.397 \\ \text { C48 } & 1.92 & 1.589 & -2.605 \\ \text { C49 } & 2.285 & 0.242 & -2.747 \\ \text { C50 } & 3.257 & -0.125 & -3.683 \\ \text { O56 } & -0.895 & 4.669 & -1.212 \\ \text { C57 } & -1.677 & 4.424 & -2.374 \\ \text { H40 } & 2.139 & 0.442 & 3.833 \\ \text { H41 } & 2.442 & 2.802 & 3.14 \\ \text { H42 } & 1.813 & 3.418 & 0.879 \\ \text { H43 } & 0.624 & -0.445 & 0.009 \\ \text { H44 } & 1.229 & -1.119 & 2.206 \\ \text { H51 } & 4.623 & 0.567 & -5.2 \\ \text { H52 } & 3.987 & 2.949 & -4.946 \\ \text { H53 } & 2.292 & 3.511 & -3.302 \\ \text { H54 } & 1.856 & -0.449 & -2.196 \\ \text { H55 } & 3.511 & -1.069 & -3.785 \\ \text { H58 } & -1.175 & 3.71 & -3.053 \\ \text { H59 } & -1.735 & 5.375 & -2.942 \\ \text { H60 } & -2.736 & 4.137 & -2.179\end{array}$

Intramolecular Epoxidation Phosphine TS

Gas Phase Energy w ZP correction: -1839.087552 Hartrees Imaginary Frequency: -4227.37

Coordinates:

$\begin{array}{lrrr}\text { C1 } & -1.2289 & 6.2965 & 0.5098 \\ \text { C2 } & -1.1154 & 4.8842 & 0.422 \\ \text { C3 } & -0.0611 & 7.1167 & 0.1295 \\ \text { C4 } & 1.2425 & 6.4338 & -0.0175 \\ \text { C5 } & 1.3352 & 5.0965 & 0.2796 \\ \text { C6 } & 0.1324 & 4.3076 & -0.3502 \\ \text { C7 } & -2.0358 & 4.1149 & 1.1715 \\ \text { C8 } & -3.148 & 4.715 & 1.7726 \\ \text { C9 } & -3.3237 & 6.0979 & 1.7052 \\ \text { C10 } & -2.3492 & 6.8931 & 1.1008 \\ \text { H11 } & -0.1161 & 8.0963 & 0.0839 \\ \text { H12 } & 2.145 & 7.0053 & -0.2099 \\ \text { H13 } & -1.889 & 3.0587 & 1.3285 \\ \text { H14 } & -3.7951 & 4.1614 & 2.2634 \\ \text { H15 } & -4.1805 & 6.5591 & 2.1809 \\ \text { H16 } & -2.4337 & 7.9707 & 1.1445 \\ \text { C17 } & -0.7976 & 1.8772 & -0.5725 \\ \text { C18 } & -0.7407 & 0.5138 & -0.1803\end{array}$




\begin{tabular}{|c|c|c|c|}
\hline C19 & 0.2968 & 2.7545 & -0.2569 \\
\hline C20 & 1.5583 & 2.1126 & -0.0127 \\
\hline C21 & 1.573 & 0.7803 & 0.4366 \\
\hline C22 & 0.421 & 0.0103 & 0.4033 \\
\hline C23 & -1.8414 & -0.3365 & -0.3923 \\
\hline C24 & -2.9839 & 0.1374 & -1.0359 \\
\hline C25 & -3.0359 & 1.4572 & -1.4774 \\
\hline C26 & -1.956 & 2.3168 & -1.2549 \\
\hline $\mathrm{H} 27$ & -1.8081 & -1.2761 & -0.1062 \\
\hline $\mathrm{H} 28$ & -3.8256 & -0.5237 & -1.2058 \\
\hline $\mathrm{H} 29$ & -3.8374 & 1.7872 & -1.9418 \\
\hline $\mathrm{H} 30$ & -2.0315 & 3.2381 & -1.571 \\
\hline $\mathrm{H} 31$ & 0.4706 & -1.0204 & 0.7307 \\
\hline H32 & 2.489 & 0.2995 & 0.7522 \\
\hline O33 & 0.1502 & 4.5831 & -1.7544 \\
\hline O34 & -0.8886 & 5.3213 & -2.1036 \\
\hline O35 & 1.3539 & 4.9313 & 1.6844 \\
\hline C36 & 2.6863 & 4.8364 & 2.1359 \\
\hline $\mathrm{H} 37$ & 2.9983 & 3.7728 & 2.1653 \\
\hline $\mathrm{H} 38$ & 2.7315 & 5.2205 & 3.1753 \\
\hline H39 & 3.409 & 5.4336 & 1.5319 \\
\hline P40 & 2.9829 & 2.7594 & -0.5273 \\
\hline C41 & 2.6998 & 3.953 & -4.9534 \\
\hline C42 & 3.1248 & 4.8844 & -4.0011 \\
\hline C43 & 3.2207 & 4.5162 & -2.6554 \\
\hline C44 & 2.8904 & 3.2136 & -2.2591 \\
\hline C45 & 2.4639 & 2.281 & -3.2154 \\
\hline C46 & 2.3693 & 2.6519 & -4.561 \\
\hline $\mathrm{H} 47$ & 2.625 & 4.2407 & -5.9943 \\
\hline $\mathrm{H} 48$ & 3.3778 & 5.8917 & -4.3064 \\
\hline $\mathrm{H} 49$ & 3.516 & 5.1773 & -1.991 \\
\hline $\mathrm{H} 50$ & 2.2186 & 1.3688 & -2.9442 \\
\hline H51 & 2.0674 & 1.9997 & -5.2312 \\
\hline C52 & 6.7723 & 0.2636 & 0.2224 \\
\hline C53 & 6.57 & 1.4612 & 0.9148 \\
\hline C54 & 5.4146 & 2.2159 & 0.6869 \\
\hline C55 & 4.4546 & 1.7712 & -0.2317 \\
\hline C56 & 4.664 & 0.573 & -0.9312 \\
\hline C57 & 5.8204 & -0.1802 & -0.7009 \\
\hline H58 & 7.6674 & -0.3187 & 0.4005 \\
\hline H59 & 7.3097 & 1.8046 & 1.6267 \\
\hline $\mathrm{H} 60$ & 5.2823 & 3.0586 & 1.1746 \\
\hline $\mathrm{H} 61$ & 3.999 & 0.2542 & -1.5801 \\
\hline H62 & 5.965 & -1.0213 & -1.1878 \\
\hline
\end{tabular}


Oxidized Phosphine Product (Compound 7)

Gas Phase Energy w ZP correction: -1839.081836 Hartrees

Coordinates:

\begin{tabular}{lrrr} 
C1 & -3.6643 & 4.1976 & 2.1219 \\
C2 & -3.9079 & 3.1298 & 1.2328 \\
C3 & -2.518 & 5.0967 & 1.892 \\
C4 & -1.6077 & 4.8709 & 0.753 \\
C5 & -1.8602 & 3.9019 & -0.1776 \\
C6 & -3.0057 & 2.9425 & 0.0186 \\
C7 & -4.9731 & 2.2475 & 1.5028 \\
C8 & -5.788 & 2.4397 & 2.6221 \\
C9 & -5.5491 & 3.509 & 3.4873 \\
C10 & -4.4888 & 4.3847 & 3.2404 \\
H11 & -2.3066 & 5.8996 & 2.5827 \\
H12 & -0.7416 & 5.5063 & 0.6409 \\
H13 & -5.1609 & 1.4025 & 0.857 \\
H14 & -6.5985 & 1.7541 & 2.8205 \\
H15 & -6.1776 & 3.6529 & 4.3537 \\
H16 & -4.3009 & 5.1995 & 3.9239 \\
O17 & -0.8173 & 3.5058 & -1.0399 \\
O18 & -3.0889 & 4.0857 & -0.8231 \\
C19 & -0.7019 & 4.3978 & -2.128 \\
H20 & 0.2256 & 4.1537 & -2.6853 \\
H21 & -1.5552 & 4.2736 & -2.8294 \\
H22 & -0.6238 & 5.4563 & -1.7949 \\
C23 & -4.2119 & 1.5721 & -1.7625 \\
C24 & -4.266 & 0.4725 & -2.6562 \\
C25 & -3.1276 & 1.6825 & -0.8264 \\
C26 & -2.1946 & 0.5955 & -0.7251 \\
C40 & -0.9759 & -1.1592 & 0.8755 \\
C27 & -2.2909 & -0.4715 & -1.6384 \\
C28 & -3.2893 & -0.5178 & -2.5976 \\
C29 & -5.3072 & 0.3665 & -3.5974 \\
C30 & -6.314 & 1.3293 & -3.6476 \\
C31 & -6.2963 & 2.3993 & -2.7576 \\
C32 & -5.2637 & 2.5206 & -1.8234 \\
H33 & -5.3389 & -0.3905 & -4.2234 \\
H34 & -7.1148 & 1.2417 & -4.372 \\
H35 & -7.0135 & 3.0715 & -2.7855 \\
H36 & -5.3016 & 3.275 & -1.1992 \\
\hline & -3.3171 & -1.357 & -3.2811 \\
H3 & -1.5778 & -1.2883 & -1.6198 \\
H3 & & & -1.1927
\end{tabular}




$\begin{array}{lrrr}\text { C42 } & 1.9846 & 1.3303 & -2.0306 \\ \text { C43 } & 0.695 & 1.1084 & -1.5382 \\ \text { C44 } & 0.4967 & 0.7566 & -0.1869 \\ \text { C45 } & 1.6244 & 0.58 & 0.6381 \\ \text { C46 } & 2.9119 & 0.8023 & 0.1388 \\ \text { H47 } & 4.0879 & 1.3536 & -1.5785 \\ \text { H48 } & 2.127 & 1.6142 & -3.0656 \\ \text { H49 } & -0.0621 & 1.2107 & -2.1548 \\ \text { H50 } & 1.5203 & 0.3023 & 1.5761 \\ \text { H51 } & 3.693 & 0.6876 & 0.724 \\ \text { C52 } & -1.6758 & 2.5031 & 4.4936 \\ \text { C53 } & -0.6506 & 2.9515 & 3.6571 \\ \text { C54 } & -0.459 & 2.3614 & 2.4037 \\ \text { C55 } & -1.2994 & 1.3204 & 1.9756 \\ \text { C56 } & -2.3153 & 0.8636 & 2.8295 \\ \text { C57 } & -2.5072 & 1.4587 & 4.0809 \\ \text { H58 } & -1.8262 & 2.9664 & 5.4602 \\ \text { H59 } & -0.0079 & 3.7613 & 3.9779 \\ \text { H60 } & 0.2627 & 2.6912 & 1.826 \\ \text { H61 } & -2.899 & 0.1231 & 2.5517 \\ \text { H62 } & -3.2271 & 1.1433 & 4.6706\end{array}$

